\title{
EQUILIBRIUM POINTS AND CENTRAL CONFIGURATIONS FOR THE LENNARD-JONES 2- AND 3-BODY PROBLEMS
}

\author{
MONTSERRAT CORBERA \\ Departament d'Informàtica i Matemàtica, Escola Politècnica Superior, Universitat \\ de Vic, C/ Laura 13, 08500 Vic, Barcelona, Spain, \\ e-mail: montserrat.corbera@uvic.es
}

JAUME LLIBRE

Departament de Matemàtiques, Universitat Autònoma de Barcelona, 08193

Bellaterra, Barcelona, Spain, e-mail: jllibre@manwe.mat.uab.es

ERNESTO PÉREZ-CHAVELA

Departamento de Matemáticas, Universidad Autónoma Metropolitana-I, Apdo. Postal 55-534,09340 México D.F., México.e-mail: epc@xanum.uam.mx

\begin{abstract}
In this paper we study the relative equilibria and their stability for a system of three point particles moving under the action of a Lennard-Jones potential. A central configuration is a special position of the particles where the position and acceleration vectors of each particle are proportional, and the constant of proportionality is the same for all particles. Since the Lennard-Jones potential depends only on the mutual distances among the particles, it is invariant under rotations. In a rotating frame the orbits coming from central configurations become equilibrium points, the relative equilibria. Due to the form of the potential, the relative equilibria depend on the size of the system, that is, depend strongly of the momentum of inertia $I$. In this work we characterize the relative equilibria, we find the bifurcation values of $I$ for which the number of relative equilibria is changing, we also analyze the stability of the relative equilibria.
\end{abstract}

Keywords: Central configurations, relative equilibria, spectral stability.

\section{Introduction}

In order to get an accurate model to study the action of the intermolecular and gravitational forces at the same time, many authors from physics, astrophysics, astronomy and chemistry have introduced new kinds of potentials, with a structure different from the classical Newtonian's and Coulombian's potentials. In this way, one potential that has been used very often in those branches of the science is the Lennard-Jones potential, which is the topic studied in this paper. For instance, it is used to model the nature and stability of small clusters of interacting particles in crystal growth, random geometry of liquids and in the theory of homogeneous nucleation, see (Hoare and Pal, 1971) and 
(Wales and Doye, 1997). This potential also appears in molecular dynamics to simulate many particle systems ranging from solids, liquids, gases, and biomolecules on Earth. Also it appears in the study of the motion of stars and galaxies in the Universe among others applications.

Most of the results about the Lennard-Jones potential are numerical, and from a static viewpoint. Here we are interested in the dynamics, we study some particular circular periodic orbits, the relative equilibria in the Lennard--Jones 2- and 3-body problems, our main contribution is the analysis of these periodic orbits, the dynamics around them and the relationship with the momentum of inertia $I$. These results can be useful to study dynamical properties such as heat transport, or relaxation of systems close to an equilibrium, until now as we know, this has been studied only far from equilibrium.

The configuration of the $N$ particles in a relative equilibrium solution remains invariant under a convenient rotation. This configuration is central and we will see that this is equivalent to say that a configuration is central if the position and acceleration vectors of each particle are proportional with the same constant of proportionality. In other words, to know relative equilibria is equivalent to know central configurations for the Lennard-Jones $N$-body problem in the plane.

This work has a wide analytical part and a short numerical one. Thus, in the analytical part for the Lennard-Jones 2- and 3-body problems we find the equilibrium points, the equilateral collinear and triangular central configurations. Moreover we study the relative equilibria associated to them and their stability. We also give sufficient conditions for the existence of isosceles collinear and triangular central configurations. In the numerical part we study the stability for the relative equilibria associated to the isosceles central configurations.

\section{Equations of motion and general aspects}

We consider $N$ particles with equal mass $m$, moving in the 2 -dimensional Euclidean space. The forces between two particles are given by a Lennard-Jones potential energy. Let $\mathbf{q}_{i} \in \mathbb{R}^{2}$ denote the position of the particle $i$ in an inertial coordinate system and let $\mathbf{q}=\left(\mathbf{q}_{1}, \ldots, \mathbf{q}_{N}\right)$. Choosing the units of mass, length and time conveniently we can think that $m=1$ and we can use the scaled Lennard-Jones pair potential:

$$
U=\sum_{1 \leqslant i<j \leqslant N}\left(\frac{1}{r_{i j}^{12}}-\frac{2}{r_{i j}^{6}}\right),
$$

where $r_{i j}=\left|\mathbf{q}_{i}-\mathbf{q}_{j}\right|$ is the distance between the particles $i$ and $j$. 
The Newton's equations of motion associated to potential (1) are given by

$$
\ddot{\mathbf{q}}=-\nabla U(\mathbf{q})
$$

or equivalently,

$$
\ddot{\mathbf{q}}_{i}=\sum_{j=1, j \neq i}^{N} 12\left(\mathbf{q}_{i}-\mathbf{q}_{j}\right)\left(\frac{1}{r_{i j}^{14}}-\frac{1}{r_{i j}^{8}}\right), \quad(i=1, \ldots, N)
$$

where the dot denotes derivative with respect to the time $t$. Equations (3) are only defined on the configuration space $\triangle=\left\{\left(\mathbf{q}_{1}, \ldots, \mathbf{q}_{N}\right) \in\right.$ $\left.\mathbb{R}^{2 N}: \mathbf{q}_{i} \neq \mathbf{q}_{j}, i \neq j\right\}$.

The center of mass of the $N$ particles is

$$
\mathbf{R}=\frac{1}{N} \sum_{i=1}^{N} \mathbf{q}_{i} .
$$

Adding the $N$ equations (3) we have that $\sum_{i=1}^{N} \ddot{\mathbf{q}}_{i}=0$. Integrating twice this equation we get $\sum_{i=1}^{N} \mathbf{q}_{i}=\mathbf{a} t+\mathbf{b}$, where $\mathbf{a}$ and $\mathbf{b}$ are constant vectors. This means that the center of mass moves, with respect to the inertial coordinate system of reference, in a straight line with constant speed.

We take the constant vectors $\mathbf{a}$ and $\mathbf{b}$ equal to zero, or equivalently, we assume that the center of mass of the particles is fixed at the origin. Then, we have the four first integrals

$$
\sum_{i=1}^{N} \mathbf{q}_{i}=0, \quad \sum_{i=1}^{N} \dot{\mathbf{q}}_{i}=0,
$$

which are known as the first integrals of the center of mass and linear momentum, respectively.

The simplest solutions are the equilibrium points; that is, when the $N$ particles are at rest for all $t \in \mathbb{R}$. Then, an equilibrium point is a solution satisfying equation

$$
\nabla U(\mathbf{q})=\mathbf{0} .
$$

Another simple type of solutions are the relative equilibrium; that is, solutions of (2) that become equilibrium points in a uniformly rotating coordinate system. These solutions are characterized as follows.

Let $R(\theta)$ denote the $2 N \times 2 N$ block diagonal matrix with $N$ blocks of size $2 \times 2$ of the form

$$
A(\theta)=\left(\begin{array}{rr}
\cos \theta & -\sin \theta \\
\sin \theta & \cos \theta
\end{array}\right) .
$$


Let $\mathbf{x} \in\left(\mathbb{R}^{2}\right)^{N}$ be a configuration of the $N$ particles, and let $\mathbf{q}(t)=$ $R(\omega t) \mathbf{x}$, where the constant $\omega$ is the angular velocity of the uniform rotating coordinate system. In the coordinate system $\mathbf{x}$ the equation of motion (2) becomes

$$
\ddot{\mathbf{x}}+2 \omega J \dot{\mathbf{x}}=-\nabla U(\mathbf{x})+\omega^{2} \mathbf{x},
$$

where $J$ is the $2 N \times 2 N$ block diagonal matrix with $N$ blocks of size $2 \times 2$ of the form

$$
\left(\begin{array}{rr}
0 & -1 \\
1 & 0
\end{array}\right) \text {. }
$$

A configuration $\mathbf{x}$ is central for system (2) if and only if $\mathbf{x}$ is an equilibrium point of system (5). That is, if and only if

$$
-\nabla U(\mathbf{x})+\omega^{2} \mathbf{x}=\mathbf{0},
$$

for some $\omega$. If $\mathbf{x}$ is a central configuration, then $\mathbf{q}(t)=R(\omega t) \mathbf{x}$ is a relative equilibrium solution of system (2). Note that $\mathbf{q}(t)=R(\omega t) \mathbf{x}$ is a periodic solution of system (2) with period $T=2 \pi /|\omega|$.

We remark that equation (6) for a central configuration $\mathbf{q}=\mathbf{x}$, says that a central configuration in the space $\mathbf{q}$ is a configuration of the particles for which the particle $\mathbf{q}$ and the acceleration $\ddot{\mathbf{q}}$ vectors of each particle are proportional, with the same constant of proportionality $\omega^{2}$.

We say that two central configurations $\mathbf{x}$ and $\mathbf{y}$ of $\left(\mathbb{R}^{2}\right)^{N}$ are related if there exist a rotation $A(\theta) \in S O(2)$ such that $R(\theta) \mathbf{x}=\mathbf{y}$, see (4). This relation is of equivalence and all the central configurations that are related to $\mathbf{x}$ are the class of equivalence of $\mathbf{x}$.

We note that in other planar problems, like for instance the planar $N$-body problem (see for a definition (Meyer and Hall, 1991)), the set of all central configurations is invariant under homotheties and rotations, while in the Lennard-Jones $N$-body problem this set is only invariant under rotations.

The study of central configurations can be seen as a problem of Lagrange multipliers where we are looking for critical points of the potential $U$ on the sphere $\left\{\mathbf{x} \in \triangle:(1 / 2) \mathbf{x}^{T} \mathbf{x}=I\right\}$ where $I>0$ is a constant. Thus $\mathbf{x}$ is a central configuration if it is a solution of system

$$
\nabla F(\mathbf{x})=\mathbf{0}, \quad i(\mathbf{x})-I=0
$$

where $F(\mathbf{x})=-U(\mathbf{x})+\omega^{2}(i(\mathbf{x})-I)$ and $i(\mathbf{x})=(1 / 2) \mathbf{x}^{T} \mathbf{x}$ is the moment of inertia.

In this paper we are also interested in the study of the stability of the relative equilibria. We will give necessary conditions for the stability or instability of the relative equilibria of the planar Lennard-Jones $N$ body problem. These conditions are based on the definitions of stability 
for the planar Newtonian $N$-body problem (see for instance (Moeckel, 1995) and (Moeckel, 1994)).

Introducing the momenta $\mathbf{p}=\dot{\mathbf{q}}$, the equations of motion (2) can be written as the Hamiltonian system

$$
\dot{\mathbf{q}}=\frac{\partial H}{\partial \mathbf{p}}=\mathbf{p}, \quad \dot{\mathbf{p}}=-\frac{\partial H}{\partial \mathbf{q}}=-\nabla U(\mathbf{q}),
$$

with Hamiltonian $H(\mathbf{q}, \mathbf{p})=\frac{1}{2} \mathbf{p}^{T} \mathbf{p}+U(\mathbf{q})$. Doing the symplectic change of variables $\mathbf{q}(t)=R(\omega t) \mathbf{x}, \mathbf{y}(t)=R^{-1}(\omega t) \mathbf{p}$ system (8) becomes the Hamiltonian system

$$
\dot{\mathbf{x}}=\frac{\partial \widetilde{H}}{\partial \mathbf{y}}=\mathbf{y}-\omega J \mathbf{x}, \quad \dot{\mathbf{y}}=-\frac{\partial \widetilde{H}}{\partial \mathbf{x}}=-\nabla U(\mathbf{x})-\omega J \mathbf{y}
$$

with Hamiltonian $\widetilde{H}(\mathbf{x}, \mathbf{y})=\frac{1}{2} \mathbf{y}^{T} \mathbf{y}+\omega \mathbf{x}^{T} J \mathbf{y}+U(\mathbf{x})$. Since an equilibrium point of (9) is given by $\mathbf{y}=\omega J \mathbf{x}$ and $-\nabla U(\mathbf{x})+\omega^{2} \mathbf{x}=\mathbf{0}$, from (6) it follows that the equilibrium points $(\mathbf{x}, \mathbf{y})$ of $(9)$ correspond to the central configurations $\mathbf{x}$ of (2).

We consider the linearization of system (9) at an equilibrium point $(\mathbf{x}, \mathbf{y})$; i.e.

$$
\dot{z}=A z
$$

where

$$
A=\left(\begin{array}{cc}
-\omega J & I d \\
-D \nabla U(\mathbf{x}) & -\omega J
\end{array}\right),
$$

and $I d$ is the $N \times N$ identity matrix.

An equilibrium point of (9) is called linearly stable if the origin of the linearized equations (10) is a stable equilibrium point. Due to the symmetries and integrals of system (9) this condition is never satisfied for the linearized equations (10), so the classical definition of linear stability does not work in the Hamiltonian case. The definition of a linearly stable relative equilibrium for the Newtonian $N$-body problem can be found, for instance, in (Moeckel, 1995) and (Moeckel, 1994).

In this work we will consider only the spectral stability, see (Moeckel, 1995) for the definition of spectral stability for the Newtonian $N$ body problem. A relative equilibrium $\mathbf{x}$ is called spectrally stable if the eigenvalues of $A$ are zero or purely imaginary.

We note that the spectral stability is a necessary condition for the stability of a relative equilibrium (see for instance (Arnold, 1978) for the definition of stability and instability of a periodic orbit) but it is not a sufficient condition. Nevertheless, if a relative equilibrium is not spectrally stable, then we know that it is unstable. 
Let $\mathbf{v}=\left(\mathbf{v}_{1}, \mathbf{v}_{2}\right)$ be an eigenvector of $A$ with eigenvalue $\lambda$. Then equation $A \mathbf{v}=\lambda \mathbf{v}$ reduces to

$$
\mathbf{v}_{2}=(\omega J+\lambda I d) \mathbf{v}_{1}, \quad B \mathbf{v}_{1}=0,
$$

where

$$
B=-D \nabla U(\mathbf{x})+\left(\omega^{2}-\lambda^{2}\right) I d-2 \lambda \omega J .
$$

Therefore, the characteristic polynomial of the matrix $A$ can be reduced to $P(\lambda)=\operatorname{det}(B)$.

\section{Lennard-Jones 2-body problem}

From (2) the equations of motion for the Lennard-Jones 2-body problem are

$$
\ddot{\mathbf{q}}=-\nabla U(\mathbf{q}),
$$

where $\mathbf{q}=\left(\mathbf{q}_{1}, \mathbf{q}_{2}\right)$ with $\mathbf{q}_{1}, \mathbf{q}_{2} \in \mathbb{R}^{2}$ and $U=\frac{1}{r^{12}}-\frac{2}{r^{6}}$, with $r=$ $\left|\mathbf{q}_{1}-\mathbf{q}_{2}\right|$.

\subsection{Equilibrium POINTS}

An equilibrium point of (12) is a solution of $\nabla U(\mathbf{q})=0$. We note that $U$ depends on $\mathbf{q}$ through the distance $r=\sqrt{\left(q_{11}-q_{21}\right)^{2}+\left(q_{12}-q_{22}\right)^{2}}$, thus we can consider $U=U(r)$. Let

$$
A=\left(\frac{\partial r}{\partial q_{11}}, \frac{\partial r}{\partial q_{12}}, \frac{\partial r}{\partial q_{21}}, \frac{\partial r}{\partial q_{22}}\right)^{T} .
$$

Using Proposition 14 (see the Appendix) we have that if the $\operatorname{rank}(A)=$ 1 , then

$$
\nabla U(\mathbf{q})=0 \quad \text { if and only if } \quad \nabla U(r)=0 .
$$

In the configuration space $\triangle, \operatorname{rank}(A)=1$. On the other hand,

$$
\nabla U(r)=-12\left(\frac{1}{r^{13}}-\frac{1}{r^{7}}\right) .
$$

Since $r \in \mathbb{R}^{+}=(0, \infty)$, the unique solution of equation $\nabla U(r)=0$ is $r=1$. Remember that we have assumed that the origin is fixed at the center of mass of the particles (i.e. $\mathbf{q}_{2}=-\mathbf{q}_{1}$ ). Therefore the equilibrium points of (12) are

$$
\left\{\left(\mathbf{q}_{1}, \mathbf{q}_{2}\right) \in \mathbb{R}^{2} \times \mathbb{R}^{2}: \mathbf{q}_{2}=-\mathbf{q}_{1},\left|\mathbf{q}_{1}-\mathbf{q}_{2}\right|=1\right\} .
$$


In short we have infinitely many equilibrium points for the LennardJones 2-body problem, one for each pair of opposite positions on the circle of radius $1 / 2$ centered at the center of mass of the two particles. In particular, Theorem 1 follows.

THEOREM 1. The equilibrium points of the Lennard-Jones 2-body problem are given by the set $\left\{\left(\mathbf{q}_{1}, \mathbf{q}_{2}\right) \in \mathbb{R}^{2} \times \mathbb{R}^{2}: \mathbf{q}_{2}=-\mathbf{q}_{1},\left|\mathbf{q}_{1}-\mathbf{q}_{2}\right|=\right.$ $1\}$.

As we will see below, the equilibrium points given by Theorem 1 are minima of the potential energy $U(\mathbf{q})$. This says, in some sense, that these equilibrium points are stable although the classical definition of stability of an equilibrium point cannot be applied in this case (see for instance (Guckenheimer and Holmes, 1986) for the definition of stable equilibrium point). This is due to the fact that we have non-isolated equilibrium points, moreover as we will see later on there are infinitely many periodic orbits as close as we want to the circle of equilibrium points.

It is easy to see that $r=1$ is a minimum of $U=\frac{1}{r^{12}}-\frac{2}{r^{6}}$, moreover at $r=1$ we have that $U=-1$. Consequently, the equilibrium points $\left\{\left(\mathbf{q}_{1}, \mathbf{q}_{2}\right) \in \mathbb{R}^{2} \times \mathbb{R}^{2}: \mathbf{q}_{2}=-\mathbf{q}_{1},\left|\mathbf{q}_{1}-\mathbf{q}_{2}\right|=1\right\}$ are minima of the potential energy, moreover at all these points the potential energy is $U=-1$.

\subsection{Central configurations}

We define the new coordinate vector $\mathbf{x}=\left(\mathbf{x}_{1}, \mathbf{x}_{2}\right)$, with $\mathbf{x}_{1}, \mathbf{x}_{2} \in \mathbb{R}^{2}$, by $\mathbf{q}(t)=R(\omega t) \mathbf{x}$ where $\omega$ is a constant (see Section 2 for details). We recall that $\mathbf{x}$ is a central configuration if it satisfies equations (7) with $U=\frac{1}{r^{12}}-\frac{2}{r^{6}}$ and $r=\left|\mathbf{x}_{1}-\mathbf{x}_{2}\right|$.

Since the center of mass is at the origin of coordinates, then $i(\mathbf{x})$ can we written as

$$
i(\mathbf{x})=\frac{1}{4} r^{2} .
$$

Therefore we can think that $F$ in (7) depends on $\mathbf{x}$ through the distance $r$. Since we do not consider collision solutions, we can apply Proposition 14, and we see that

$$
\nabla F(\mathbf{x})=0 \quad \text { if and only if } \quad \frac{\mathrm{d} F}{\mathrm{~d} r}=0 .
$$

In short, equations (7) can be written as

$$
12\left(\frac{1}{r^{13}}-\frac{1}{r^{7}}\right)+\frac{1}{2} r \omega^{2}=0, \quad \frac{1}{4} r^{2}=I .
$$


Solving the second equation of (13) we have that $r=2 \sqrt{I}$. Substituting $r$ into the first equation of (13), and solving the resulting equation with respect to $\omega$, we obtain

$$
\omega= \pm \frac{\sqrt{3}}{16} \sqrt{-\frac{1}{8 I^{7}}+\frac{8}{I^{4}}} .
$$

This is only possible when $-\frac{1}{8 I^{7}}+\frac{8}{I^{4}} \geqslant 0$. The solutions of this inequality are $I \in\left[\frac{1}{4}, \infty\right)$. If $I=\frac{1}{4}$, then $\omega=0$ and we obtain the equilibrium point that we have found in Section 3.1. For each value of $I \in\left(\frac{1}{4}, \infty\right)$ we have a central configuration with $r=2 \sqrt{I}$. For $I \in\left(0, \frac{1}{4}\right)$ we have no central configurations. In short, we have proved the following result.

PROPOSITION 2. For each value of $I \in\left(\frac{1}{4}, \infty\right)$ we have the central configurations $\left\{\left(\mathbf{x}_{1}, \mathbf{x}_{2}\right) \in \mathbb{R}^{2} \times \mathbb{R}^{2}: \mathbf{x}_{2}=-\mathbf{x}_{1},\left|\mathbf{x}_{1}-\mathbf{x}_{2}\right|=2 \sqrt{I}\right\}$.

\section{THEOREM 3.}

(a) Let $\left(\mathbf{x}_{1}, \mathbf{x}_{2}\right)$ be a central configuration, for a fixed value of $I \in$ $\left(\frac{1}{4}, \infty\right)$, given by Proposition 2. Then $\left(\mathbf{x}_{1}, \mathbf{x}_{2}\right)$ provides a relative equilibrium solution of the Lennard-Jones 2-body problem with period $T=2 \pi /|\omega|$ which is given by

$\mathbf{q}_{1}(t)=\sqrt{I}(\cos (\omega t), \sin (\omega t)), \quad \mathbf{q}_{2}(t)=\sqrt{I}(-\cos (\omega t),-\sin (\omega t))$,

where

$$
\omega= \pm \frac{\sqrt{3}}{16} \sqrt{-\frac{1}{8 I^{7}}+\frac{8}{I^{4}}}
$$

(b) The period $T$ as a function of $I$ is plotted in Figure 1 and it satisfies the following properties:

(i) $\lim _{I \rightarrow 1 / 4} T(I)=\infty$.

(ii) $\lim _{I \rightarrow \infty} T(I)=\infty$.

(iii) $T(I)$ has a minimum at the point $I_{0}=\frac{1}{4}\left(\frac{7}{4}\right)^{1 / 3}$ with $T\left(I_{0}\right)=$ $\frac{7 \pi}{6}\left(\frac{7}{32}\right)^{1 / 6}$

(iv) $T(I)$ decreases in $\left(1 / 4, I_{0}\right)$ and increases in $\left(I_{0}, \infty\right)$. 


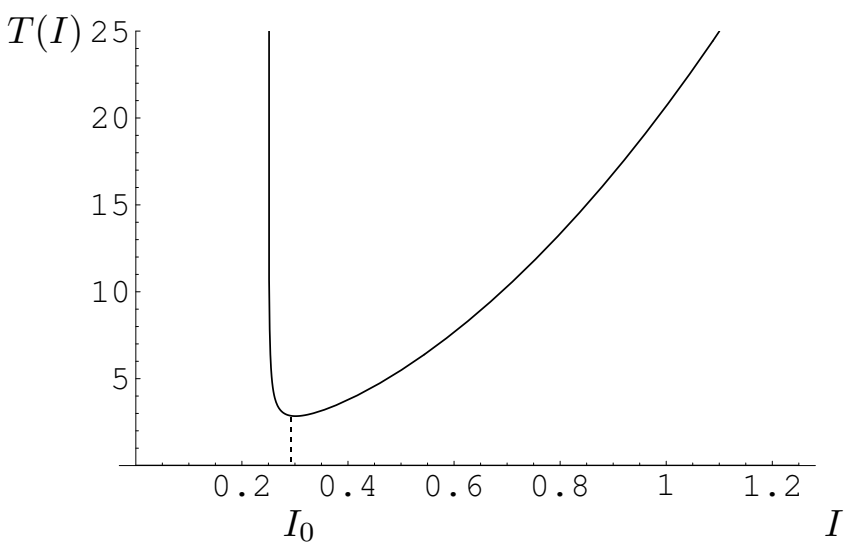

Figure 1. Plot of $T(I)$.

Proof: The proof of statement (a) follows immediately from the definition of relative equilibrium solutions.

Statement (b) is obtained by analyzing the properties of the period function $T=2 \pi /|\omega|$.

\subsection{SpeCtral Stability of the CEntral CONFigurations}

Now we analyze the spectral stability of the relative equilibria of the Lennard-Jones 2-body problem given by Proposition 2 .

Let $\mathbf{x}=\left(\mathbf{x}_{1}, \mathbf{x}_{2}\right)$, with $\mathbf{x}_{1}=(\sqrt{I}, 0)$ and $\mathbf{x}_{2}=(-\sqrt{I}, 0)$ and $I \in$ $\left(\frac{1}{4}, \infty\right)$, be a representant of the class of central configurations given in Proposition 2. The relative equilibrium associated to $\mathbf{x}$ is spectrally stable if the $\lambda$ 's satisfying

$$
\operatorname{det}(B)=\operatorname{det}\left[-D \nabla U(\mathbf{x})+\left(\omega^{2}-\lambda^{2}\right) I d-2 \lambda \omega J\right]=0,
$$

are zero or purely imaginary (see Section 2 for details). Here $U(\mathbf{x})=$ $\frac{1}{r^{12}}-\frac{2}{r^{6}}, r=\left|\mathbf{x}_{1}-\mathbf{x}_{2}\right|, I d$ is the $4 \times 4$ identity matrix, and $\omega=$ $\pm \frac{\sqrt{3}}{16} \sqrt{-\frac{1}{8 I^{7}}+\frac{8}{I^{4}}}$.

After some computations we see that the solutions of equation (14) are

$$
\begin{aligned}
& \lambda=0, \text { with multiplicity } 2, \\
& \lambda= \pm \omega i, \quad \text { with multiplicity } 2, \\
& \lambda= \pm \frac{\sqrt{3} \sqrt{-5+128 I^{3}}}{32 I^{7 / 2}}
\end{aligned}
$$


Analyzing the sign of $f(I)=-5+128 I^{3}$ for $I \in\left(\frac{1}{4}, \infty\right)$, we see that $f(I)<0$ if $I \in\left(\frac{1}{4}, \frac{1}{4}\left(\frac{5}{2}\right)^{1 / 3}\right), f(I)=0$ if $I=\frac{1}{4}\left(\frac{5}{2}\right)^{1 / 3}$ and $f(I)>0$ if $I \in\left(\frac{1}{4}\left(\frac{5}{2}\right)^{1 / 3}, \infty\right)$. In short we have proved the following result.

PROPOSITION 4. The relative equilibria of the Lennard-Jones 2body problem (given by Theorem 3) are unstable for $I>\frac{1}{4}\left(\frac{5}{2}\right)^{1 / 3}$ and spectrally stable for $I \in\left(\frac{1}{4}, \frac{1}{4}\left(\frac{5}{2}\right)^{1 / 3}\right]$.

\section{Lennard-Jones 3-body problem}

From (2) the equations of motion for the Lennard-Jones 3-body problem are

$$
\ddot{\mathbf{q}}=-\nabla U(\mathbf{q}),
$$

where $\mathbf{q}=\left(\mathbf{q}_{1}, \mathbf{q}_{2}, \mathbf{q}_{3}\right), \mathbf{q}_{i} \in \mathbb{R}^{2}$,

$$
U(\mathbf{q})=\frac{1}{r_{12}^{12}}-\frac{2}{r_{12}^{6}}+\frac{1}{r_{13}^{12}}-\frac{2}{r_{13}^{6}}+\frac{1}{r_{23}^{12}}-\frac{2}{r_{23}^{6}},
$$

and $r_{i j}=\left|\mathbf{q}_{i}-\mathbf{q}_{j}\right|$.

\subsection{EQUILIBRIUM POINTS}

An equilibrium point of system (15) is a solution of

$$
\nabla U(\mathbf{q})=0 \text {. }
$$

We note that $U$ depends on $\mathbf{q}$ by means of the mutual distances $r_{i j}$, thus we can consider $U=U\left(r_{12}, r_{13}, r_{23}\right)$ where $\left(r_{12}, r_{13}, r_{23}\right)=$ $\left(r_{12}\left(q_{11}, q_{12}, q_{21}, q_{22}\right), r_{13}\left(q_{11}, q_{12}, q_{31}, q_{32}\right), r_{23}\left(q_{21}, q_{22}, q_{31}, q_{32}\right)\right)$. Using Proposition 14, we have that if $\operatorname{rank}(A)=3$, with $A$ given by

$$
\left(\begin{array}{lll}
\frac{\partial r_{12}}{\partial q_{11}} & \frac{\partial r_{13}}{\partial q_{11}} & \frac{\partial r_{23}}{\partial q_{11}} \\
\frac{\partial r_{12}}{\partial q_{12}} & \frac{\partial r_{13}}{\partial q_{12}} & \frac{\partial r_{23}}{\partial q_{12}} \\
\frac{\partial r_{12}}{\partial q_{21}} & \frac{\partial r_{13}}{\partial q_{21}} & \frac{\partial r_{23}}{\partial q_{21}} \\
\frac{\partial r_{12}}{\partial q_{22}} & \frac{\partial r_{13}}{\partial q_{22}} & \frac{\partial r_{23}}{\partial q_{22}} \\
\frac{\partial r_{12}}{\partial q_{31}} & \frac{\partial r_{13}}{\partial q_{31}} & \frac{\partial r_{23}}{\partial q_{31}} \\
\frac{\partial r_{12}}{\partial q_{32}} & \frac{\partial r_{13}}{\partial q_{32}} & \frac{\partial r_{23}}{\partial q_{32}}
\end{array}\right)=\left(\begin{array}{ccc}
\frac{q_{11}-q_{21}}{r_{12}} & \frac{q_{11}-q_{31}}{r_{13}} & 0 \\
\frac{q_{12}-q_{22}}{r_{12}} & \frac{q_{12}-q_{32}}{r_{13}} & 0 \\
-\frac{q_{11}-q_{21}}{r_{12}} & 0 & \frac{q_{21}-q_{31}}{r_{23}} \\
-\frac{q_{12}-q_{22}}{r_{12}} & 0 & \frac{q_{22}-q_{32}}{r_{23}} \\
0 & -\frac{q_{11}-q_{31}}{r_{13}} & -\frac{q_{21}-q_{31}}{r_{23}} \\
0 & -\frac{q_{12}-q_{32}}{r_{13}} & -\frac{q_{22}-q_{32}}{r_{23}}
\end{array}\right),
$$


then

$$
\nabla U(\mathbf{q})=0 \quad \text { if and only if } \quad \nabla U\left(r_{12}, r_{13}, r_{23}\right)=0 .
$$

After some computations we see that $\operatorname{rank}(A)=3$ if and only if

$$
\operatorname{det}\left(\begin{array}{lll}
q_{11} & q_{12} & 1 \\
q_{21} & q_{22} & 1 \\
q_{31} & q_{32} & 1
\end{array}\right) \neq 0
$$

This determinant equals twice the area of the triangle formed by the 3 bodies. In short, we have proved:

LEMMA 5. If $\mathbf{q}_{1}, \mathbf{q}_{2}$ and $\mathbf{q}_{3}$ are not collinear, then $\nabla U\left(r_{12}, r_{13}, r_{23}\right)=$ $\mathbf{0}$ if and only if $\nabla U(\mathbf{q})=\mathbf{0}$.

In order to find the equilibrium points of the Lennard-Jones 3-body problem we will distinguish two problems, the collinear and the planar Lennard-Jones 3-body problem. In the first case the three particles move on a straight line, so we cannot find the equilibrium points by solving directly $\nabla U=\mathbf{0}$ through the mutual distances. In this case we will find the equilibrium points by using the position variables q. We note that we could use the mutual distances if we found the critical points of the function $U\left(r_{12}, r_{13}, r_{23}\right)$ on the curve $r_{13}=r_{12}+r_{23}$ by means of Lagrange multipliers (this fact can be proved using similar arguments to the ones given in Proposition 14).

In particular we will prove the following result.

\section{THEOREM 6.}

(a) The collinear equilibrium points of the Lennard-Jones 3-body problem are given by the sets

$$
\begin{aligned}
& \left\{\left(\mathbf{q}_{1}, \mathbf{q}_{2}, \mathbf{q}_{3}\right) \in \mathbb{R}^{6}: \mathbf{q}_{2}=\mathbf{0},\left|\mathbf{q}_{1}-\mathbf{q}_{3}\right|=a, \mathbf{q}_{3}=-\mathbf{q}_{1}-\mathbf{q}_{2}\right\}, \\
& \left\{\left(\mathbf{q}_{1}, \mathbf{q}_{2}, \mathbf{q}_{3}\right) \in \mathbb{R}^{6}: \mathbf{q}_{1}=\mathbf{0},\left|\mathbf{q}_{2}-\mathbf{q}_{3}\right|=a, \mathbf{q}_{3}=-\mathbf{q}_{1}-\mathbf{q}_{2}\right\}, \\
& \left\{\left(\mathbf{q}_{1}, \mathbf{q}_{2}, \mathbf{q}_{3}\right) \in \mathbb{R}^{6}: \mathbf{q}_{3}=\mathbf{0},\left|\mathbf{q}_{1}-\mathbf{q}_{2}\right|=a, \mathbf{q}_{3}=-\mathbf{q}_{1}-\mathbf{q}_{2}\right\},
\end{aligned}
$$

where $a=\left(\frac{2731}{43}\right)^{1 / 6}$.

(b) The planar non-collinear equilibrium points of the LennardJones 3-body problem are given by the set

$$
\begin{aligned}
\left\{\left(\mathbf{q}_{1}, \mathbf{q}_{2}, \mathbf{q}_{3}\right) \in \mathbb{R}^{6}: \quad\right. & \left|\mathbf{q}_{1}-\mathbf{q}_{2}\right|=\left|\mathbf{q}_{1}-\mathbf{q}_{3}\right|=\left|\mathbf{q}_{2}-\mathbf{q}_{3}\right|=1, \\
& \left.\mathbf{q}_{3}=-\mathbf{q}_{1}-\mathbf{q}_{2} .\right\}
\end{aligned}
$$


The proof of statement (a) (respectively (b)) of Theorem 6 is given in Subsection 4.1.1 (respectively 4.1.2).

\subsubsection{Collinear equilibrium points}

Now we consider the collinear Lennard-Jones 3-body problem. Here, $\mathbf{q}_{1}=q_{1}, \mathbf{q}_{2}=q_{2}, \mathbf{q}_{3}=q_{3} \in \mathbb{R}$. Since the origin is taken at the center of mass of the particles, we have that $q_{3}=-q_{1}-q_{2}$. Then, the equations of motion (15) can be written as

$$
\begin{aligned}
& \ddot{q}_{1}=12\left(\frac{1}{\left(q_{1}-q_{2}\right)^{13}}-\frac{1}{\left(q_{1}-q_{2}\right)^{7}}+\frac{1}{\left(2 q_{1}+q_{2}\right)^{13}}-\frac{1}{\left(2 q_{1}+q_{2}\right)^{7}}\right), \\
& \ddot{q}_{2}=12\left(-\frac{1}{\left(q_{1}-q_{2}\right)^{13}}+\frac{1}{\left(q_{1}-q_{2}\right)^{7}}+\frac{1}{\left(q_{1}+2 q_{2}\right)^{13}}-\frac{1}{\left(q_{1}+2 q_{2}\right)^{7}}\right) .
\end{aligned}
$$

We note that the third equation of (15) has been omitted because it is a linear combination of the first two equations.

Let $a_{12}=q_{1}-q_{2}, a_{13}=q_{1}-q_{3}=2 q_{1}+q_{2}$ and $a_{23}=q_{2}-q_{3}=q_{1}+2 q_{2}$. Then, an equilibrium point of (18) is a solution of system

$$
\frac{1}{a_{12}^{13}}-\frac{1}{a_{12}^{7}}+\frac{1}{a_{13}^{13}}-\frac{1}{a_{13}^{7}}=0, \quad-\frac{1}{a_{12}^{13}}+\frac{1}{a_{12}^{7}}+\frac{1}{a_{23}^{13}}-\frac{1}{a_{23}^{7}}=0 .
$$

Without loss of generality we can choose the order of the particles in such a way that $a_{12}>0, a_{13}>0$ and $a_{23}>0$. Then, it is easy to see that $a_{12}, a_{13}$ and $a_{23}$ must satisfy

$$
a_{13}=a_{12}+a_{23} .
$$

In short we must solve the system

$$
\begin{aligned}
& \frac{1}{a_{12}^{13}}-\frac{1}{a_{12}^{7}}+\frac{1}{\left(a_{12}+a_{23}\right)^{13}}-\frac{1}{\left(a_{12}+a_{23}\right)^{7}}=0, \\
& -\frac{1}{a_{12}^{13}}+\frac{1}{a_{12}^{7}}+\frac{1}{a_{23}^{13}}-\frac{1}{a_{23}^{7}}=0 .
\end{aligned}
$$

After factorizing the two equations of (21), we see that system (21) is equivalent to the system

$$
\left(2 a_{12}+a_{23}\right) f_{1}\left(a_{12}, a_{23}\right)=0, \quad\left(a_{12}-a_{23}\right) f_{2}\left(a_{12}, a_{23}\right)=0,
$$

where $f_{1}\left(a_{12}, a_{23}\right)$ is

$$
\begin{aligned}
& -a_{12}^{12}+a_{12}^{18}-6 a_{12}^{11} a_{23}+9 a_{12}^{17} a_{23}-36 a_{12}^{10} a_{23}^{2}+42 a_{12}^{16} a_{23}^{2}-125 a_{12}^{9} a_{23}^{3}+ \\
& 132 a_{12}^{15} a_{23}^{3}-295 a_{12}^{8} a_{23}^{4}+299 a_{12}^{14} a_{23}^{4}-496 a_{12}^{7} a_{23}^{5}+497 a_{12}^{13} a_{23}^{5}-610 a_{12}^{6} a_{23}^{6}+ \\
& 610 a_{12}^{12} a_{23}^{6}-553 a_{12}^{5} a_{23}^{7}+553 a_{12}^{11} a_{23}^{7}-367 a_{12}^{4} a_{23}^{8}+367 a_{12}^{10} a_{23}^{8}-174 a_{12}^{3} a_{23}^{9}+ \\
& 174 a_{12}^{9} a_{23}^{9}-56 a_{12}^{2} a_{23}^{10}+56 a_{12}^{8} a_{23}^{10}-11 a_{12} a_{23}^{11}+11 a_{12}^{7} a_{23}^{11}-a_{23}^{12}+a_{12}^{6} a_{23}^{12},
\end{aligned}
$$


and $f_{2}\left(a_{12}, a_{23}\right)$ is

$$
\begin{aligned}
& -a_{12}^{12}-a_{12}^{11} a_{23}-a_{12}^{10} a_{23}^{2}-a_{12}^{9} a_{23}^{3}-a_{12}^{8} a_{23}^{4}-a_{12}^{7} a_{23}^{5}-a_{12}^{6} a_{23}^{6}+a_{12}^{12} a_{23}^{6}- \\
& a_{12}^{5} a_{23}^{7}+a_{12}^{11} a_{23}^{7}-a_{12}^{4} a_{23}^{8}+a_{12}^{10} a_{23}^{8}-a_{12}^{3} a_{23}^{9}+a_{12}^{9} a_{23}^{9}-a_{12}^{2} a_{23}^{10}+ \\
& a_{12}^{8} a_{23}^{10}-a_{12} a_{23}^{11}+a_{12}^{7} a_{23}^{11}-a_{23}^{12}+a_{12}^{6} a_{23}^{12} .
\end{aligned}
$$

Then, the solutions of (22) must satisfy one of the following four sets of conditions

$$
\begin{array}{ll}
a_{12}=-a_{23} / 2, & a_{12}=a_{23}, \\
a_{12}=-a_{23} / 2, & f_{2}\left(a_{12}, a_{23}\right)=0, \\
a_{12}=a_{23}, & f_{1}\left(a_{12}, a_{23}\right)=0, \\
f_{1}\left(a_{12}, a_{23}\right)=0, & f_{2}\left(a_{12}, a_{23}\right)=0 .
\end{array}
$$

Since we have assumed that $a_{i j}>0$, conditions (23) and (24) are not satisfied. Solving $f_{1}\left(a_{12}, a_{23}\right)=0$ with $a_{12}=a_{23}$, we obtain the solution

$$
a_{12}=a_{23}=\frac{1}{2}\left(\frac{2731}{43}\right)^{1 / 6}, \quad a_{13}=\left(\frac{2731}{43}\right)^{1 / 6} .
$$

In order to solve system (26), we have computed the resultant of $f_{1}\left(a_{12}\right.$, $\left.a_{23}\right)$ and $f_{2}\left(a_{12}, a_{23}\right)$ with respect to $a_{12}$ (respectively, $\left.a_{23}\right)$ obtaining a polynomial $P\left(a_{23}\right)$ (respectively, $Q\left(a_{12}\right)$ ). The different combinations of a root of $Q\left(a_{12}\right)$ with a root of $P\left(a_{23}\right)$ give us the possible solutions of system (26). We note that we are only interested in positive real solutions. After some computations we see that there is no combination of a positive real root of $Q\left(a_{12}\right)$ with a positive real root of $P\left(a_{23}\right)$ satisfying (26). Therefore condition (26) never is satisfied.

We have just seen that if we choose the order of the particles in such a way that $q_{1}-q_{2}>0, q_{1}-q_{3}>0$ and $q_{2}-q_{3}>0$ (this means that the order of the particles on the straight line is $m_{1}, m_{2}$ and $m_{3}$ from right to left), then there is a unique equilibrium point of the collinear Lennard--Jones 3-body problem which is given by

$$
\begin{aligned}
\left\{\left(q_{1}, q_{2}, q_{3}\right) \in \mathbb{R}^{3}: \quad\right. & q_{1}-q_{2}=q_{2}-q_{3}=a / 2, q_{1}-q_{3}=a, \\
& \left.q_{3}=-q_{1}-q_{2}\right\},
\end{aligned}
$$

or equivalently, $\left(q_{1}, q_{2}, q_{3}\right)=(-a / 2,0, a / 2)$ where $a=\left(\frac{2731}{43}\right)^{1 / 6}$.

We are interested in equilibrium points of the Lennard--Jones 3 body problem (15). It is easy to see that the equilibrium point of the collinear Lennard-Jones 3-body problem $\left(q_{1}, q_{2}, q_{3}\right)=(-a / 2,0, a / 2)$ gives infinitely many equilibrium points of (15), one for each straight line passing through the origin. For all these equilibrium points the 
particle $m_{2}$ is placed at the origin and the other two particles are placed at the ends of a diameter of the circle of radius $a / 2$ centered at the origin. In short the equilibrium point of the collinear Lennard-Jones 3 -body problem $\left(q_{1}, q_{2}, q_{3}\right)=(-a / 2,0, a / 2)$ provides infinitely many equilibrium points of the Lennard Jones 3 -body problem which are given by the set

$$
\left\{\left(\mathbf{q}_{1}, \mathbf{q}_{2}, \mathbf{q}_{3}\right) \in \mathbb{R}^{6}: \mathbf{q}_{2}=\mathbf{0},\left|\mathbf{q}_{1}-\mathbf{q}_{3}\right|=a, \mathbf{q}_{3}=-\mathbf{q}_{1}-\mathbf{q}_{2}\right\},
$$

or equivalently,

$$
\left(\mathbf{q}_{1}, \mathbf{q}_{2}, \mathbf{q}_{3}\right)=(a / 2 \cos \theta, a / 2 \sin \theta, 0,0,-a / 2 \cos \theta,-a / 2 \sin \theta),
$$

with $\theta \in[0,2 \pi)$. Notice that we have a continuum of equilibrium points parameterized by $\theta$.

We can chose five other possible orders for the particles in the collinear Lennard-Jones 3-body problem. Analyzing these other possible choices we obtain exactly two other different sets of equilibrium points of the Lennard Jones 3 -body problem. They are given by

$$
\left\{\left(\mathbf{q}_{1}, \mathbf{q}_{2}, \mathbf{q}_{3}\right) \in \mathbb{R}^{6}: \mathbf{q}_{1}=\mathbf{0},\left|\mathbf{q}_{2}-\mathbf{q}_{3}\right|=a, \mathbf{q}_{3}=-\mathbf{q}_{1}-\mathbf{q}_{2}\right\},
$$

and

$$
\left\{\left(\mathbf{q}_{1}, \mathbf{q}_{2}, \mathbf{q}_{3}\right) \in \mathbb{R}^{6}: \mathbf{q}_{3}=\mathbf{0},\left|\mathbf{q}_{1}-\mathbf{q}_{2}\right|=a, \mathbf{q}_{3}=-\mathbf{q}_{1}-\mathbf{q}_{2}\right\}
$$

In short we have proved statement (a) of Theorem 6 .

Let $M\left(\mathbf{q}_{1}, \mathbf{q}_{2}, \mathbf{q}_{3}\right)$ be the Hessian matrix of the potential energy $U(\mathbf{q})$ given by (16). It is not difficult to see that the eigenvalues of $M$ at the collinear equilibrium points of the Lennard-Jones 3-body given by $(27),(28)$ and $(29)$ are

$$
\begin{aligned}
\lambda_{1} & =0, \text { with multiplicity } 3 & \lambda_{2} & =\frac{6601761792}{7458361}\left(\frac{43}{2731}\right)^{1 / 3}, \\
\lambda_{3} & =\frac{798768}{2731}\left(\frac{43}{2731}\right)^{1 / 3}, & \lambda_{4} & =-\frac{8322048}{7458361}\left(\frac{43}{2731}\right)^{1 / 3} .
\end{aligned}
$$

Since $M$ has positive and negative eigenvalues, the collinear equilibrium points of the Lennard-Jones 3-body problem are unstable. 


\subsubsection{Planar equilibrium points}

In order to find planar equilibrium points of the Lennard-Jones 3-body problem which are not collinear, it is sufficient to solve equation

$$
\nabla U\left(r_{12}, r_{13}, r_{23}\right)=-12\left(\begin{array}{c}
r_{12}^{-13}-r_{12}^{-7} \\
r_{13}^{-13}-r_{13}^{-7} \\
r_{23}^{-13}-r_{23}^{-7}
\end{array}\right)=\left(\begin{array}{l}
0 \\
0 \\
0
\end{array}\right) \text {. }
$$

We solve the equation $\frac{1}{r^{13}}-\frac{1}{r^{7}}=0$ obtaining a unique positive real root $r=1$. So, $\nabla U\left(r_{12}, r_{13}, r_{23}\right)=\mathbf{0}$ if and only if $r_{12}=r_{13}=r_{23}=1$. Therefore we have infinitely many planar equilibrium points of the Lennard-Jones 3-body problem which are characterized by the set

$$
\begin{aligned}
\left\{\left(\mathbf{q}_{1}, \mathbf{q}_{2}, \mathbf{q}_{3}\right) \in \mathbb{R}^{6}: \quad\right. & \left|\mathbf{q}_{1}-\mathbf{q}_{2}\right|=\left|\mathbf{q}_{1}-\mathbf{q}_{3}\right|=\left|\mathbf{q}_{2}-\mathbf{q}_{3}\right|=1, \\
& \left.\mathbf{q}_{3}=-\mathbf{q}_{1}-\mathbf{q}_{2}\right\}
\end{aligned}
$$

which proves statement (b) of Theorem 6 .

We note that for the planar equilibrium points of the Lennard--Jones 3 -body problem, the three particles form an equilateral triangle of side 1 whose barycenter is the center of mass. In particular, it is not difficult to see that the planar equilibrium points are

$$
\left(\mathbf{q}_{1}, \mathbf{q}_{2}, \mathbf{q}_{3}\right)=(1 / \sqrt{3})\left(\cos \theta, \sin \theta, \cos \theta_{1}, \sin \theta_{1}, \cos \theta_{2}, \sin \theta_{2}\right),
$$

and

$$
\left(\mathbf{q}_{1}, \mathbf{q}_{2}, \mathbf{q}_{3}\right)=(1 / \sqrt{3})\left(\cos \theta, \sin \theta, \cos \theta_{2}, \sin \theta_{2}, \cos \theta_{1}, \sin \theta_{1}\right),
$$

where $\theta_{1}=\theta+\frac{2 \pi}{3}, \theta_{2}=\theta+\frac{4 \pi}{3}$ and $\theta \in[0,2 \pi)$.

Now we shall see that the planar equilibrium points (30) are minima of the potential energy $U(\mathbf{q})$ (which is given in (16)).

We consider the potential energy as a function of the mutual distances $r_{i j}$; i.e. $U=U\left(r_{12}, r_{13}, r_{23}\right)$. We have just seen that $r_{12}=r_{13}=$ $r_{23}=1$ is a critical point of $U\left(r_{12}, r_{13}, r_{23}\right)$. Let $M\left(r_{12}, r_{13}, r_{23}\right)$ be the Hessian matrix of $U\left(r_{12}, r_{13}, r_{23}\right)$. After some computations we see that the eigenvalues of $M$ at $r_{12}=r_{13}=r_{23}=1$ are $\lambda=72$ with multiplicity 3. Therefore $r_{12}=r_{13}=r_{23}=1$ is a minimum of $U\left(r_{12}, r_{13}, r_{23}\right)$, moreover $U(1,1,1)=-3$. Since the potential energy $U(\mathbf{q})$ depends on $\mathbf{q}$ through the mutual distances $r_{i j}=\left|\mathbf{q}_{i}-\mathbf{q}_{j}\right|$, it follows that the points of the set (30) are minima of the potential $U(\mathbf{q})$. Moreover on the set (30) the potential energy equals -3 . 


\subsection{Central configurations}

We define the new coordinate vector $\mathbf{x}=\left(\mathbf{x}_{1}, \mathbf{x}_{2}, \mathbf{x}_{3}\right)$ with $\mathbf{x}_{1}, \mathbf{x}_{2}, \mathbf{x}_{3} \in$ $\mathbb{R}^{2}$ by $\mathbf{q}(t)=R(\omega t) \mathbf{x}$ where $\omega$ is a constant (see Section 2 for details). We recall that $\mathbf{x}$ is a central configuration if it satisfies system (7) with

$$
U(\mathbf{x})=\frac{1}{r_{12}^{12}}-\frac{2}{r_{12}^{6}}+\frac{1}{r_{13}^{12}}-\frac{2}{r_{13}^{6}}+\frac{1}{r_{23}^{12}}-\frac{2}{r_{23}^{6}},
$$

where $r_{i j}=\left|\mathbf{x}_{i}-\mathbf{x}_{j}\right|$.

We note that $U$ depends on $\mathbf{x}$ by means of the mutual distances $r_{i j}$. Since we have chosen the origin of the coordinates at the center of mass of $m_{1}, m_{2}$ and $m_{3}$, then $i(\mathbf{x})$ can we written as

$$
i(\mathbf{x})=(1 / 6)\left(r_{12}^{2}+r_{13}^{2}+r_{23}^{2}\right) .
$$

Therefore we can think that $F$ depends on $\mathbf{x}$ through the mutual distances $r_{i j}$.

Working as in Subsection 4.1 we can see that if $\mathbf{x}_{1}, \mathbf{x}_{2}$ and $\mathbf{x}_{3}$ are not collinear, then $\nabla F\left(r_{12}, r_{13}, r_{23}\right)=\mathbf{0}$ if and only if $\nabla F(\mathbf{q})=\mathbf{0}$. Thus in order to find the central configurations of the Lennard-Jones 3-body problem we also will distinguish two problems, the collinear and the planar Lennard-Jones 3-body problem. In particular we will prove the following result which is a summary of the future Lemmas 9 and 10 . In the next result we only provide the geometry of the central configurations for the planar Lennard-Jones 3-body problem.

THEOREM 7. The planar Lennard-Jones 3-body problem has the following geometrical central configurations (see Figure 2).

(a) For $I \in\left(0, I_{1}\right]$ with $I_{1}=1 / 2$ there are no central configurations.

(b) For any $I>I_{1}$ there is a unique equilateral triangle central configuration (i.e. the three particles are at the vertices of an equilateral triangle).

(c) When $I=I_{2}$ with $I_{2}=0.595186 \ldots$ an isosceles triangle central configuration appears which bifurcates for $I>I_{2}$ in two different isosceles triangle central configurations (denoted by $T_{1}$ and $T_{2}$ ).

(i) The isosceles triangle central configuration $T_{1}$ has the two equal sides smaller than the third one for all $I_{2} \leqslant I<I_{5}$ with $I_{5}=1.001285 \ldots$. When $I=I_{5}, T_{1}$ degenerates to a segment; i.e. it becomes a collinear central configuration which coincides with the collinear central configuration given in statement(d). Finally, there is no isosceles triangle central configuration $T_{1}$ when $I>I_{5}$. 


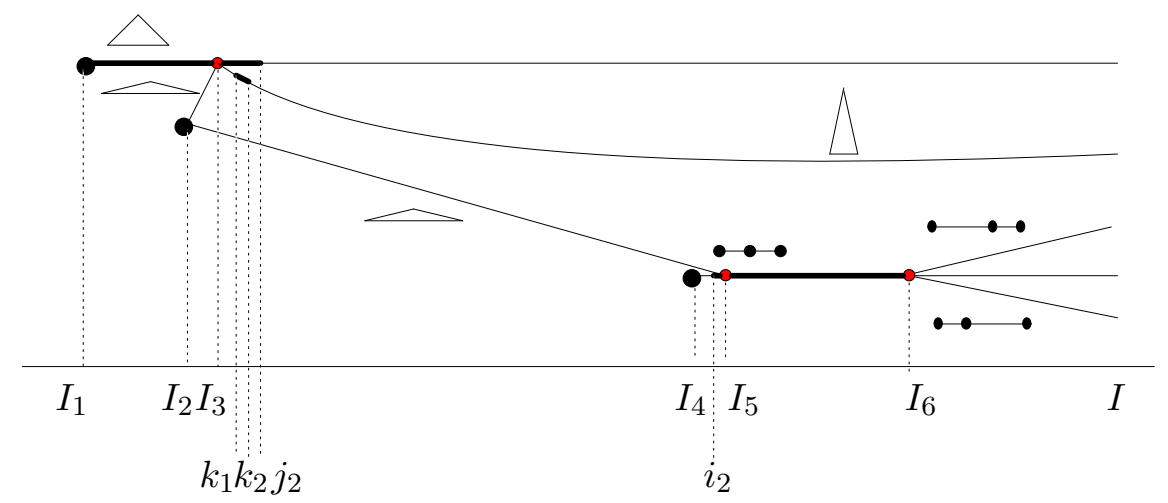

Figure 2. The different central configurations depending on the moment of inertia $I$.

(ii) The isosceles triangle central configuration $T_{2}$ has the two equal sides smaller than the third one for $I \in\left(I_{2}, I_{3}\right)$ with $I_{3}=\frac{1}{2}\left(\frac{7}{4}\right)^{1 / 3}=0.602535 \ldots$. At $I=I_{3}$ it coincides with the equilateral triangle central configuration given in statement (b). Finally, when $I>I_{3}, T_{2}$ is an isosceles triangle where the two equal sides are larger than the third one.

(d) For $I>I_{4}$ with $I_{4}=\frac{1}{4}\left(\frac{2731}{43}\right)^{1 / 3}=0.99745 \ldots$ there is an additionally equilateral collinear central configuration (i.e. the three particles are on a straight line and one of the particles is located exactly at the middle point of the segment formed by the other two).

(e) When $I>I_{6}$ with $I_{6}=\frac{1}{4}\left(\frac{36409}{313}\right)^{1 / 3}=1.22038 \ldots$ we have two isosceles collinear central configurations (i.e. the three particles are on a straight line and one of the particles is located inside the segment formed by the other two but not exactly at the middle point) that bifurcate form the equilateral collinear central configuration for $I=I_{6}$.

Taking into account all the different distributions of the particles in the geometrical central configurations described in Theorem 7 we obtain the following corollary.

COROLLARY 8. The number of classes of central configurations of the planar Lennard-Jones 3-body problem for the different values of the moment of inertia I are summarized in Table $I$. 
Table I. Number of central configurations for the planar Lennard--Jones 3-body problem.

\begin{tabular}{c|c}
\hline $\mathrm{I}$ & $\#$ \\
\hline$\left(0, I_{1}\right]$ & 0 \\
$\left(I_{1}, I_{2}\right)$ & 2 \\
$I_{2}$ & 8
\end{tabular}

\begin{tabular}{c|r}
\hline $\mathrm{I}$ & $\#$ \\
\hline$\left(I_{2}, I_{3}\right)$ & 14 \\
$I_{3}$ & 8 \\
$\left(I_{3}, I_{4}\right]$ & 14
\end{tabular}

\begin{tabular}{c|c}
\hline $\mathrm{I}$ & $\#$ \\
\hline$\left(I_{4}, I_{5}\right)$ & 17 \\
{$\left[I_{5}, I_{6}\right]$} & 11 \\
$\left(I_{6}, \infty\right)$ & 17
\end{tabular}

\subsubsection{Collinear central configurations}

In order to find the collinear central configurations for the LennardJones 3-body problem we cannot solve directly $\nabla F=\mathbf{0}$ through the mutual distances $r_{i j}$ (see Lemma 5), thus in this case we will solve directly system (7) with $U$ given by (31). Similarly to the collinear equilibrium points, if we use the mutual distances in order to find the collinear central configurations, then we find the critical points of the function $U\left(r_{12}, r_{13}, r_{23}\right)$ on $\left\{\left(r_{12}, r_{13}, r_{23}\right) \in \mathbb{R}^{+} \times \mathbb{R}^{+} \times \mathbb{R}^{+}: \frac{1}{6}\left(r_{12}^{2}+\right.\right.$ $\left.\left.r_{13}^{2}+r_{23}^{2}\right)=I, r_{13}=r_{12}+r_{23}\right\}$ by means of the Lagrange multipliers.

Suppose that $\mathbf{x}_{1}=x_{1}, \mathbf{x}_{2}=x_{2}, \mathbf{x}_{3}=x_{3} \in \mathbb{R}$. Since we have taken the origin at the center of mass of the particles (i.e. $x_{3}=-x_{1}-x_{2}$ ), we can write the moment of inertia as in (32). Therefore, (7) becomes

$$
\nabla F(\mathbf{x})=\mathbf{0}, \quad i(\mathbf{x})-I=0
$$

where $F(\mathbf{x})=-U(\mathbf{x})+\omega^{2}(i(\mathbf{x})-I), i(\mathbf{x})=\frac{1}{6}\left(r_{12}^{2}+r_{13}^{2}+r_{23}^{2}\right), U(\mathbf{x})$ is given by (31) and $r_{i j}=\left|x_{i}-x_{j}\right|$.

Let $a_{12}=x_{1}-x_{2}, a_{13}=x_{1}-x_{3}=2 x_{1}+x_{2}$ and $a_{23}=x_{2}-x_{3}=$ $x_{1}+2 x_{2}$. As in Subsection 4.1.1, we can choose the order of the masses in such a way that $a_{12}>0, a_{13}>0$ and $a_{23}>0$. Then, system (33) becomes

$$
\begin{aligned}
12\left(\frac{1}{a_{12}^{13}}-\frac{1}{a_{12}^{7}}\right)+12\left(\frac{1}{a_{13}^{13}}-\frac{1}{a_{13}^{7}}\right)+\frac{1}{3} \omega^{2}\left(a_{12}+a_{13}\right) & =0, \\
-12\left(\frac{1}{a_{12}^{13}}-\frac{1}{a_{12}^{7}}\right)+12\left(\frac{1}{a_{23}^{13}}-\frac{1}{a_{23}^{7}}\right)+\frac{1}{3} \omega^{2}\left(-a_{12}+a_{23}\right) & =0, \\
-12\left(\frac{1}{a_{13}^{13}}-\frac{1}{a_{13}^{7}}\right)-12\left(\frac{1}{a_{23}^{13}}-\frac{1}{a_{23}^{7}}\right)+\frac{1}{3} \omega^{2}\left(-a_{13}-a_{23}\right) & =0, \\
\frac{1}{6}\left(a_{12}^{2}+a_{13}^{2}+a_{23}^{2}\right) & =I .
\end{aligned}
$$

It is easy to see that the second equation of (34) is a linear combination of the first and the third equations, so we eliminate it. Since we have assumed that $a_{i j}>0$, then from the first and the third equations 
of (34) we have

$$
\begin{aligned}
\omega^{2} & =-\frac{36}{a_{12}+a_{13}}\left(\frac{1}{a_{12}^{13}}-\frac{1}{a_{12}^{7}}+\frac{1}{a_{13}^{13}}-\frac{1}{a_{13}^{7}}\right) \\
& =-\frac{36}{a_{13}+a_{23}}\left(\frac{1}{a_{13}^{13}}-\frac{1}{a_{13}^{7}}+\frac{1}{a_{23}^{13}}-\frac{1}{a_{23}^{7}}\right) .
\end{aligned}
$$

Therefore, we shall solve system

$$
\begin{aligned}
& \frac{1}{a_{12}+a_{13}}\left(\frac{1}{a_{12}^{13}}-\frac{1}{a_{12}^{7}}+\frac{1}{a_{13}^{13}}-\frac{1}{a_{13}^{7}}\right)=\frac{1}{a_{13}+a_{23}}\left(\frac{1}{a_{13}^{13}}-\frac{1}{a_{13}^{7}}+\frac{1}{a_{23}^{13}}-\frac{1}{a_{23}^{7}}\right), \\
& a_{12}^{2}+a_{13}^{2}+a_{23}^{2}=6 I, \\
& a_{13}=a_{12}+a_{23} .
\end{aligned}
$$

Factorizing the first equation of (36), after the substitution $a_{13}=$ $a_{12}+a_{23}$, we have that it is equivalent to the equation

$$
\left(-a_{12}+a_{23}\right) f\left(a_{12}, a_{23}\right)=0 .
$$

where $f\left(a_{12}, a_{23}\right)$ is

$$
\begin{aligned}
& -a_{12}{ }^{24}-12 a_{12}{ }^{23} a_{23}-68 a_{12}{ }^{22} a_{23}{ }^{2}-242 a_{12}{ }^{21} a_{23}{ }^{3}-609 a_{12}{ }^{20} a_{23}{ }^{4}- \\
& 1162 a_{12}{ }^{19} a_{23}{ }^{5}-1772 a_{12}{ }^{18} a_{23}{ }^{6}+a_{12}{ }^{24} a_{23}{ }^{6}-2268 a_{12}{ }^{17} a_{23}{ }^{7}+ \\
& 12 a_{12}{ }^{23} a_{23}{ }^{7}-2563 a_{12}{ }^{16} a_{23}{ }^{8}+68 a_{12}{ }^{22} a_{23}{ }^{8}-2688 a_{12}{ }^{15} a_{23}{ }^{9}+ \\
& 242 a_{12}{ }^{21} a_{23}{ }^{9}-2724 a_{12}{ }^{14} a_{23}{ }^{10}+609 a_{12}{ }^{20} a_{23}{ }^{10}-2730 a_{12}{ }^{13} a_{23}{ }^{11}+ \\
& 1162 a_{12}{ }^{19} a_{23}{ }^{11}-2731 a_{12}{ }^{12} a_{23}{ }^{12}+1772 a_{12}{ }^{18} a_{23}{ }^{12}-2730 a_{12}{ }^{11} a_{23}{ }^{13}+ \\
& 2268 a_{12}{ }^{17} a_{23}{ }^{13}-2724 a_{12}{ }^{10} a_{23}{ }^{14}+2558 a_{12}{ }^{16} a_{23}{ }^{14}-2688 a_{12}{ }^{9} a_{23}{ }^{15}+ \\
& 2648 a_{12}{ }^{15} a_{23}{ }^{15}-2563 a_{12}{ }^{8} a_{23}{ }^{16}+2558 a_{12}{ }^{14} a_{23}{ }^{16}-2268 a_{12}{ }^{7} a_{23}{ }^{17}+ \\
& 2268 a_{12}{ }^{13} a_{23}{ }^{17}-1772 a_{12}{ }^{6} a_{23}{ }^{18}+1772 a_{12}{ }^{12} a_{23}{ }^{18}-1162 a_{12}{ }^{5} a_{23}{ }^{19}+ \\
& 1162 a_{12}{ }^{11} a_{23}{ }^{19}-609 a_{12}{ }^{4} a_{23}{ }^{20}+609 a_{12}{ }^{10} a_{23}{ }^{20}-242 a_{12}{ }^{3} a_{23}{ }^{21}+ \\
& 242 a_{12}{ }^{9} a_{23}{ }^{21}-68 a_{12}{ }^{2} a_{23}{ }^{22}+68 a_{12}{ }^{8} a_{23}{ }^{22}-12 a_{12} a_{23}{ }^{23}+ \\
& 12 a_{12}{ }^{7} a_{23}{ }^{23}-a_{23}{ }^{24}+a_{12}{ }^{6} a_{23}{ }^{24} \text {. }
\end{aligned}
$$

Then, the first equation of (36) is satisfied if either $a_{12}=a_{23}$ or $f\left(a_{12}, a_{23}\right)=0$.

If $a_{12}=a_{23}$, then the second equation of system (36) has a unique positive solution $a_{23}=\sqrt{I}$. Therefore, $a_{12}=\sqrt{I}$, and consequently, $a_{13}=2 \sqrt{I}$. So, $\left(a_{12}, a_{13}, a_{23}\right)=(\sqrt{I}, 2 \sqrt{I}, \sqrt{I})$ is a solution of (36). This solution must satisfy that $\omega^{2}>0$. Substituting it into (35), we see that this is only possible when $I \in\left(I_{4}, \infty\right)$ where $I_{4}=\frac{1}{4}\left(\frac{2731}{43}\right)^{1 / 3}=$ $0.99745 \ldots$ 
Now we must analyze the solutions of system

$$
\begin{aligned}
f\left(a_{12}, a_{23}\right) & =0 \\
a_{12}^{2}+\left(a_{12}+a_{23}\right)^{2}+a_{23}^{2} & =6 I .
\end{aligned}
$$

Solving the second equation of (37) we have that $a_{12}=\frac{1}{2}\left(-a_{23} \pm\right.$ $\left.\sqrt{3} \sqrt{4 I-a_{23}^{2}}\right)$. Since $a_{12}>0$ and $a_{23}>0$, we only are interested in the solution

$$
a_{12}=\frac{1}{2}\left(-a_{23}+\sqrt{3} \sqrt{4 I-a_{23}^{2}}\right) \text { with } a_{23} \in(0, \sqrt{3 I}) .
$$

If we take $a_{12}$ and $a_{23}$ satisfying (38), then the first equation of (37) is equivalent to equation

$$
f_{1}\left(a_{23}, I\right)=0
$$

with $f_{1}\left(a_{23}, I\right)$ equal to

$$
\begin{aligned}
& -a_{23}{ }^{24}+a_{23}{ }^{30}+24 a_{23}{ }^{22} I-30 a_{23}{ }^{28} I-252 a_{23}{ }^{20} I^{2}+405 a_{23}{ }^{26} I^{2}+ \\
& 2052 a_{23}{ }^{18} I^{3}-3348 a_{23}{ }^{24} I^{3}-15390 a_{23}{ }^{16} I^{4}+19602 a_{23}{ }^{22} I^{4}+ \\
& 86508 a_{23}{ }^{14} I^{5}-88452 a_{23}{ }^{20} I^{5}-332424 a_{23}{ }^{12} I^{6}+317115 a_{23}{ }^{18} I^{6}+ \\
& 918540 a_{23}{ }^{10} I^{7}-887922 a_{23}{ }^{16} I^{7}-1896129 a_{23}{ }^{8} I^{8}+1863324 a_{23}{ }^{14} I^{8}+ \\
& 2794986 a_{23}{ }^{6} I^{9}-2794986 a_{23}{ }^{12} I^{9}-2834352 a_{23}{ }^{4} I^{10}+ \\
& 2834352 a_{23}{ }^{10} I^{10}+1771470 a_{23}{ }^{2} I^{11}-1771470 a_{23}{ }^{8} I^{11}- \\
& 531441 I^{12}+531441 a_{23}{ }^{6} I^{12} .
\end{aligned}
$$

Now we analyze the solution of (39) depending on the values of $I$. First we find the bifurcation values for the solutions of (39). These values are given by the solutions of system

$$
f_{1}\left(a_{23}, I\right)=0, \quad f_{2}\left(a_{23}, I\right)=0
$$

where

$$
f_{2}\left(a_{23}, I\right)=\frac{\partial f_{1}}{\partial a_{23}}\left(a_{23}, I\right)=6 a_{23}\left(a_{23}^{2}-3 I\right)\left(a_{23}^{2}-I\right) g\left(a_{23}, I\right),
$$

with $g\left(a_{23}, I\right)$ equal to

$$
\begin{aligned}
& -4 a_{23}{ }^{18}+5 a_{23}{ }^{24}+72 a_{23}{ }^{16} I-120 a_{23}{ }^{22} I-540 a_{23}{ }^{14} I^{2}+ \\
& 1260 a_{23}{ }^{20} I^{2}+3780 a_{23}{ }^{12} I^{3}-7992 a_{23}{ }^{18} I^{3}-24300 a_{23}{ }^{10} I^{4}+ \\
& 36126 a_{23}{ }^{16} I^{4}+93312 a_{23}{ }^{8} I^{5}-126360 a_{23}{ }^{14} I^{5}-218700 a_{23}{ }^{6} I^{6}+ \\
& 337527 a_{23}{ }^{12} I^{6}+376164 a_{23}{ }^{4} I^{7}-638604 a_{23}{ }^{10} I^{7}-367416 a_{23}{ }^{2} I^{8}+ \\
& 780759 a_{23}{ }^{8} I^{8}+196830 I^{9}-551124 a_{23}{ }^{6} I^{9}+177147 a_{23}{ }^{4} I^{10} .
\end{aligned}
$$


Remember that we only are interested in the positive values of $a_{23}$ and $I$.

Substituting the solution $a_{23}=\sqrt{I}$ of the second equation of (40) into the first equation of (40) we get the equation

$$
I^{12}\left(-36409+20032 I^{3}\right)=0 \text {. }
$$

So $I=0$ and $I=c_{1}=\frac{1}{4}\left(\frac{36409}{313}\right)^{1 / 3}=1.22038 \ldots$ are the bifurcation values. In a similar way, substituting the solution $a_{23}=\sqrt{3 I}$ of the second equation of (40) into the first equation of (40), we have that $I=0$ is the unique bifurcation value. Finally, we solve the system

$$
f_{1}\left(a_{23}, I\right)=0, \quad g\left(a_{23}, I\right)=0,
$$

and we obtain another bifurcation value $I=c_{0}=0.0813699 \ldots$. This system has been solved by computing the resultant of $f_{1}\left(a_{23}, I\right)$ and $g\left(a_{23}, I\right)$ with respect to $I$ and with respect to $a_{23}$, and then solving the corresponding polynomials (this method has already been used in Subsection 4.1.1 in a similar case).

Solving equation (39) for different values of $I$, we see that when $I=0$ equation (39) has three real roots: $a_{23}=-1$ and $a_{23}=1$ with multiplicity one, and $a_{23}=0$ with multiplicity 24 . For $I \in\left(0, c_{0}\right)$ we have four positive real roots that bifurcate from $a_{23}=0$, and another real root that comes from the root $a_{23}=1$ (we do not consider negative real roots). When $I=c_{0}$ one of the four real roots that bifurcate from $a_{23}=0$ joins with the root that comes from $a_{23}=1$ giving a double root and we still have the other three roots that come from $a_{23}=0$. The double root disappears when $I>c_{0}$ whereas the other three roots persist. When $I=c_{1}$ appears a new double root, from which emerges two roots for $I>c_{1}$ (see for more details Figure 3).

Now we see which of these solutions of (39) really give solutions of (37). First, we need that $a_{23} \in(0, \sqrt{3 I})$. We see that $a_{23}=0$ and $a_{23}=\sqrt{3 I}$ are solutions of $f_{1}\left(a_{23}, I\right)$ only when $I=0$. Therefore, if a branch solution $a_{23}(I)$ of $(39)$ satisfies $a_{23} \in(0, \sqrt{3 I})$ for a fixed value of $I>0$, then it satisfies this condition for all $I>0$. In particular, we can see that the only two branches that satisfy condition $a_{23} \in(0, \sqrt{3 I})$ are the ones that come from the double root that appears when $I=c_{1}$.

In short, when $I<c_{1}$ we have no solutions of (37). When $I=c_{1}$ we have a unique solution which is given by

$$
a_{23}=\sqrt{I}, \quad a_{12}=\sqrt{I} .
$$

Finally when $I>c_{1}$ we have two solutions of (39) that we denote by $\alpha(I)$ and $\bar{\alpha}(I)$. It is not difficult to see that these two solutions provide 


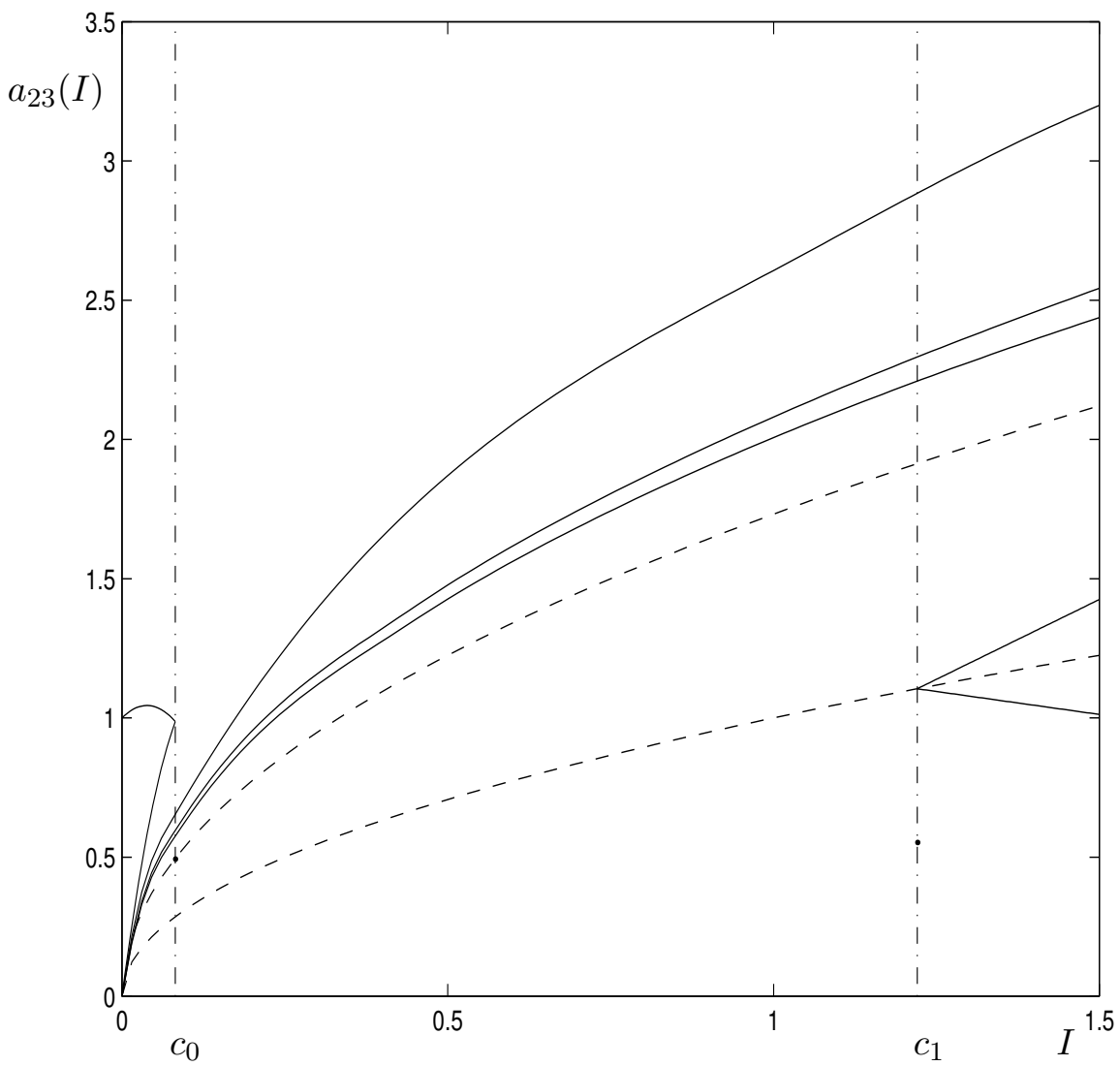

Figure 3. The bifurcation values $c_{0}$ and $c_{1}$ of $f_{1}\left(a_{23}, I\right)=0$ with respect to the parameter $I$. The upper dashed line corresponds to the curve $a_{23}(I)=\sqrt{3 I}$ and the lower dashed line corresponds to the curve $a_{23}(I)=\sqrt{I}$ (on this line we can find the equilateral collinear central configurations).

two solutions of (37) which are

$$
\left\{a_{23}=\alpha(I), a_{12}=\bar{\alpha}(I)\right\} \quad \text { and } \quad\left\{a_{23}=\bar{\alpha}(I), a_{12}=\alpha(I)\right\} .
$$

On the other hand, the solutions of (37) that we have found will provide solutions of (34) only if they satisfy $\omega^{2}>0$ where $\omega^{2}$ is given by (35). If we take $a_{12}$ and $a_{23}$ as in (38), then $\omega^{2}$ becomes

$$
\omega^{2}=\frac{2415919104 h\left(a_{23}, I\right)}{\left(-a_{23}+\sqrt{3} \sqrt{-a_{23}^{2}+4 I}\right)^{13}\left(a_{23}+\sqrt{3} \sqrt{-a_{23}{ }^{2}+4 I}\right)^{13}},
$$

with $h\left(a_{23}, I\right)$ equal to

$$
-a_{23}{ }^{12}+a_{23}{ }^{18}+24 a_{23}{ }^{10} I-30 a_{23}{ }^{16} I-162 a_{23}{ }^{8} I^{2}+378 a_{23}{ }^{14} I^{2}+
$$




$$
\begin{aligned}
& 162 a_{23}{ }^{6} I^{3}-2619 a_{23}{ }^{12} I^{3}+1620 a_{23}{ }^{4} I^{4}+10854 a_{23}{ }^{10} I^{4}- \\
& 3645 a_{23}{ }^{2} I^{5}-26973 a_{23}{ }^{5} I^{5}-729 I^{6}+36450 a_{23}{ }^{6} I^{6}-15309 a_{23}{ }^{4} I^{7}- \\
& 19683 a_{23}{ }^{2} I^{8}+19683 I^{9} .
\end{aligned}
$$

Since $a_{23} \in(0, \sqrt{3 I})$, the denominator of (41) is positive. It only remains to prove that $h\left(a_{23}, I\right)$ is positive on the solutions of (39). To do that we solve system

$$
f_{1}\left(a_{23}, I\right)=0, \quad h\left(a_{23}, I\right)=0,
$$

by computing the resultant of $f_{1}\left(a_{23}, I\right)$ and $h\left(a_{23}, I\right)$ with respect to $a_{23}$ and with respect to $I$, and finding the roots of the resulting polynomials. We see that there are no real positive solutions of (42). Therefore, $\omega^{2}$ is either always positive or always negative on the solutions of (39), in particular we see that it is always positive.

Remember that $a_{12}=x_{1}-x_{2}, a_{13}=2 x_{1}+x_{2}, a_{23}=x_{1}+2 x_{2}$, and $x_{3}=-x_{1}-x_{2}$. Thus, we get

$$
x_{1}=\frac{1}{3}\left(2 a_{12}+a_{23}\right), \quad x_{2}=\frac{1}{3}\left(-a_{12}+a_{23}\right), \quad x_{3}=\frac{1}{3}\left(-a_{12}-2 a_{23}\right) .
$$

In short, we have proved the following result.

LEMMA 9. If we choose the order of the particles in such a way that $x_{1}-x_{2}>0, x_{1}-x_{3}>0$ and $x_{2}-x_{3}>0$ (this means that the order of the particles on the straight line is $m_{1}, m_{2}$ and $m_{3}$ from right to left), then we have the following central configurations.

(a) If $I>I_{4}$ with $I_{4}=\frac{1}{4}\left(\frac{2731}{43}\right)^{1 / 3}=0.99745 \ldots$, then we have a unique collinear equilateral central configuration (i.e. the three particles are on a straight line and the separation between $m_{1}$ and $m_{2}$ is the same as that between $m_{2}$ and $m_{3}$ ) given by

$$
a_{12}=a_{23}=\sqrt{I}, \quad a_{13}=2 \sqrt{I},
$$

or equivalently,

$$
\left(x_{1}, x_{2}, x_{3}\right)=(\sqrt{I}, 0,-\sqrt{I})
$$

(b) If $I>I_{6}$ with $I_{6}=c_{1}=\frac{1}{4}\left(\frac{36409}{313}\right)^{1 / 3}=1.22038 \ldots$, then we have two collinear isosceles central configurations (i.e. the three particles are on a straight line and the separation between $m_{1}$ and $m_{2}$ is different from that between $m_{2}$ and $m_{3}$ ) satisfying

$$
\begin{array}{lll}
a_{23}=\alpha(I), & a_{12}=\bar{\alpha}(I), & a_{13}=\alpha(I)+\bar{\alpha}(I), \\
a_{23}=\bar{\alpha}(I), & a_{12}=\alpha(I), & a_{13}=\alpha(I)+\bar{\alpha}(I),
\end{array}
$$




$$
\begin{aligned}
& \text { or equivalently, } \\
& \left(x_{1}, x_{2}, x_{3}\right)=\frac{1}{3}(2 \bar{\alpha}(I)+\alpha(I),-\bar{\alpha}(I)+\alpha(I),-\bar{\alpha}(I)-2 \alpha(I)) \\
& \left(x_{1}, x_{2}, x_{3}\right)=\frac{1}{3}(2 \alpha(I)+\bar{\alpha}(I),-\alpha(I)+\bar{\alpha}(I),-\alpha(I)-2 \bar{\alpha}(I)) .
\end{aligned}
$$

Statements (d) and (e) of Theorem 7 follow from Lemma 9.

As in Subsection 4.1.1, we can choose five other possible orders for the particles in the collinear Lennard--Jones 3-body problem. Analyzing these choices we obtain different central configurations. In particular, (43) provides only two additional classes of central configurations for $I>I_{4}$, they are

$$
\left(x_{1}, x_{2}, x_{3}\right)=(0, \sqrt{I},-\sqrt{I}), \quad\left(x_{1}, x_{2}, x_{3}\right)=(\sqrt{I},-\sqrt{I}, 0) .
$$

On the other hand, (45) provides four additional classes of central configurations for $I>I_{6}$, which are given by

$$
\begin{aligned}
& \left(x_{1}, x_{2}, x_{3}\right)=(1 / 3)(2 \bar{\alpha}(I)+\alpha(I),-\bar{\alpha}(I)-2 \alpha(I),-\bar{\alpha}(I)+\alpha(I)), \\
& \left(x_{1}, x_{2}, x_{3}\right)=(1 / 3)(2 \alpha(I)+\bar{\alpha}(I),-\alpha(I)-2 \bar{\alpha}(I),-\alpha(I)+\bar{\alpha}(I)), \\
& \left(x_{1}, x_{2}, x_{3}\right)=(1 / 3)(-\bar{\alpha}(I)+\alpha(I),-\bar{\alpha}(I)-2 \alpha(I), 2 \bar{\alpha}(I)+\alpha(I)), \\
& \left(x_{1}, x_{2}, x_{3}\right)=(1 / 3)(-\alpha(I)+\bar{\alpha}(I),-\alpha(I)-2 \bar{\alpha}(I), 2 \alpha(I)+\bar{\alpha}(I)) .
\end{aligned}
$$

We remark that the three equilateral collinear central configurations for $I>I_{4}$ bifurcate at $I=I_{4}$ from the three circles of collinear equilibrium points of the Lennard-Jones 3-body problem that are given in Theorem 6(a).

\subsubsection{Planar central configurations}

In order to find the central configurations of the planar Lennard--Jones 3 -body problem which are not collinear we solve system (7) with $U$ given by (31) in terms of the mutual distances $r_{i j}$; that is, we solve the system

$$
\begin{aligned}
12\left(\frac{1}{r_{12}^{13}}-\frac{1}{r_{12}^{7}}\right)+\frac{1}{3} r_{12} \omega^{2} & =0, \\
12\left(\frac{1}{r_{13}^{13}}-\frac{1}{r_{13}^{7}}\right)+\frac{1}{3} r_{13} \omega^{2} & =0, \\
12\left(\frac{1}{r_{23}^{13}}-\frac{1}{r_{23}^{7}}\right)+\frac{1}{3} r_{23} \omega^{2} & =0, \\
\frac{1}{6}\left(r_{12}^{2}+r_{13}^{2}+r_{23}^{2}\right) & =I .
\end{aligned}
$$




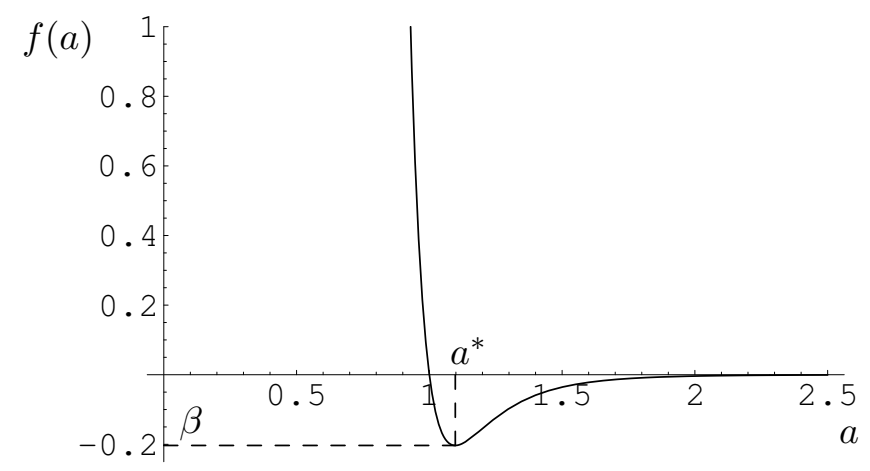

Figure 4. Plot of $f(a)$.

Using the first three equations of (46) we have that $\omega^{2}$ is given by

$$
-36\left(\frac{1}{r_{12}^{14}}-\frac{1}{r_{12}^{8}}\right)=-36\left(\frac{1}{r_{13}^{14}}-\frac{1}{r_{13}^{8}}\right)=-36\left(\frac{1}{r_{23}^{14}}-\frac{1}{r_{23}^{8}}\right) \text {. }
$$

We will solve system

$$
\begin{gathered}
\frac{1}{r_{12}^{14}}-\frac{1}{r_{12}^{8}}=\frac{1}{r_{13}^{14}}-\frac{1}{r_{13}^{8}} \\
\frac{1}{r_{12}^{14}}-\frac{1}{r_{12}^{8}}=\frac{1}{r_{23}^{14}}-\frac{1}{r_{23}^{8}} \\
r_{12}^{2}+r_{13}^{2}+r_{23}^{2}=6 I
\end{gathered}
$$

First, we analyze the first two equations of (48). Let $f(a)=\frac{1}{a^{14}}-\frac{1}{a^{8}}$. We note that $\lim _{a \rightarrow 0^{+}} f(a)=\infty, \lim _{a \rightarrow \infty} f(a)=0$ and $f(a)$ has a minimum at the point $a=a^{*}=\left(\frac{7}{4}\right)^{1 / 6}=1.09776 \ldots$ with $\beta=f\left(a^{*}\right)=$ $-\frac{12}{49}\left(\frac{4}{7}\right)^{1 / 3}=-0.203223 \ldots$ (see Figure 4$)$.

We note that the first two equations of (48) can be written as

$$
f\left(r_{12}\right)=f\left(r_{13}\right)=f\left(r_{23}\right) .
$$

Since $\omega^{2}>0$ (we do not consider the case $\omega=0$ because it corresponds to an equilibrium point of the collinear Lennard-Jones 3-body problem), we are only interested in values of $r_{i j}$ such that $f\left(r_{i j}\right)<0$ (see $(47))$. 
Table II. Different types of solutions of (49).

(i) $\quad r_{12}=r_{13}=r_{23}=a_{1}$,
(ii) $r_{12}=r_{13}=r_{23}=a_{2}$,
(iii) $\begin{cases}r_{12}=r_{13}=a_{1}, & r_{23}=a_{2} \\ r_{12}=r_{23}=a_{1}, & r_{13}=a_{2}, \\ r_{13}=r_{23}=a_{1}, & r_{12}=a_{2},\end{cases}$
(iv) $\begin{cases}r_{12}=r_{13}=a_{2}, & r_{23}=a_{1}, \\ r_{12}=r_{23}=a_{2}, & r_{13}=a_{1}, \\ r_{13}=r_{23}=a_{2}, & r_{12}=a_{1},\end{cases}$
(v) $r_{12}=r_{13}=r_{23}=a^{*}$.

Fixed a value of $c \in(\beta, 0)$, we can find two different values $a_{1} \in$ $\left(1, a^{*}\right)$ and $a_{2} \in\left(a^{*}, \infty\right)$ satisfying $f\left(a_{i}\right)=c$ (see Figure 4). From these two values, we obtain eight different solutions of (49), which are detailed in types (i)-(iv) of Table II. If $c=\beta$, then there is a unique value $a^{*}$ such that $f\left(a^{*}\right)=c$. Thus, in this case we have a unique solution of (49), which corresponds to type (v) in Table II.

On the other hand, $r_{i j}$ must verify the third equation of (48). Thus, for the solutions of types (i), (ii) and (v) we have that $r_{12}=r_{13}=r_{23}=$ $\sqrt{2 I}$. In particular, if we have a solution of type (i), then $I \in\left(I_{1}, I_{3}\right)$ with $I_{1}=1 / 2$ and $I_{3}=\left(a^{*}\right)^{2} / 2$. If we have a solution of type (ii), then $I \in\left(I_{3}, \infty\right)$; and if we have a solution of type (v), then $I=I_{3}$.

For $\beta<c<0$, we have computed numerically the values of $a_{1}$ and $a_{2}$ satisfying $f\left(a_{i}\right)=c$. Then, we have computed the values of the moment of inertia $I$ of the solutions associated to types (iii) and (iv) for these values of $a_{i}$ and we have plotted it in Figure 5. We see that the solutions of types (iv) always have $I \in\left(I_{3}, \infty\right)$. For the solutions of types (iii), $I$ has a minimum at the point $c=\xi=-0.18830 \ldots$ with $I=I_{2}=0.595186 \ldots$

We note that if $2 a_{1}=a_{2}$, then the solutions of type (iii) are in fact collinear. After doing some computations, we see that $2 a_{1}=a_{2}$ only when $I=I_{5}=1.001285 \ldots$ (or equivalently, when $c=\eta=$ $-0.0038257 \ldots$. . Therefore, for $I=I_{5}$, we obtain a collinear central configuration which correspond to the one obtained in Lemma 9(a). Moreover if $2 a_{1}<a_{2}$, then there is no possible configurations for the three particles in the plane satisfying the conditions of type (iii). We can see that if $I>I_{5}$, then $2 a_{1}<a_{2}$. Therefore we have no solutions of type (iii) for $I>I_{5}$.

Solutions of types (i), (ii) and (v) correspond to equilateral triangles and solutions (iii) and (iv) correspond to isosceles triangles.

In short, we have proved the following result. 


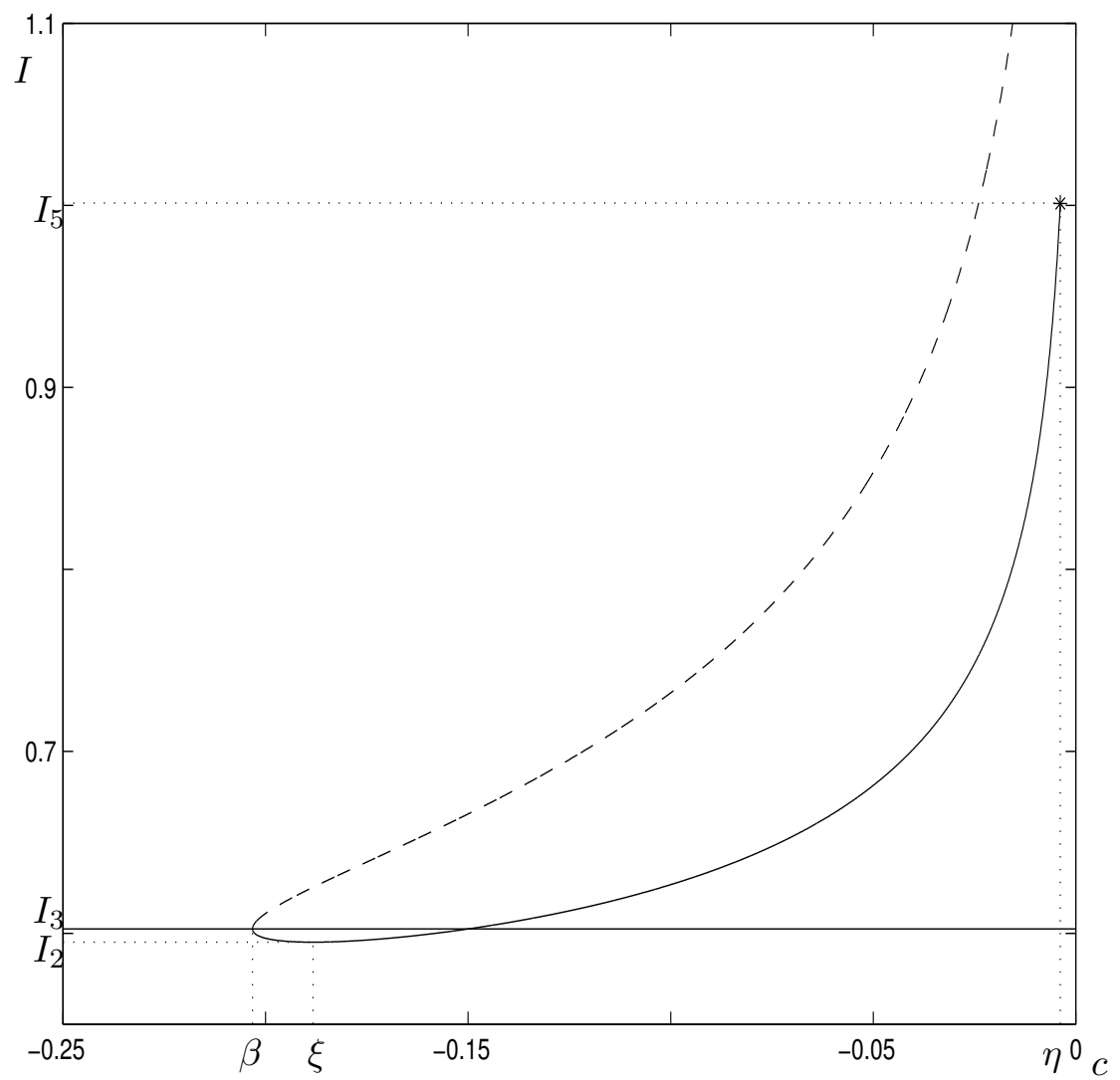

Figure 5. Plot of the moment inertia $I$ as a function of the value $c \in(\beta, 0)$. The plot with a dashed line corresponds to solutions of types (iv). The plot with continuous line correspond to solutions of types (iii).

LEMMA 10. Depending on the values of the moment of inertia I we have the following planar non-collinear central configurations of the Lennard-Jones 3-body problem.

(a) If $I \in\left(0, I_{1}\right]$, then we have no central configurations.

(b) If $I \in\left(I_{1}, I_{2}\right)$, then we have an equilateral triangle of type (i).

(c) If $I=I_{2}$, then we have an equilateral triangle of type (i) and an isosceles triangle of type (iii).

(d) If $I \in\left(I_{2}, I_{3}\right)$, then we have an equilateral triangle of type (i) and two isosceles triangles of type (iii).

(e) If $I=I_{3}$, then we have an equilateral triangle of type (v) and an isosceles triangle of type (iii). 
(f) If $I \in\left(I_{3}, I_{5}\right)$, then we have an equilateral triangle of type (ii), and two isosceles triangles of types (iii) and (iv) respectively.

(g) If $I=I_{5}$, then we have an equilateral triangle of type (ii), an isosceles triangle of type (iv) and a collinear equilateral central configuration.

(h) If $I \in\left(I_{5}, \infty\right)$, then we have an equilateral triangle of type (ii) and an isosceles triangle of type (iv).

Statements (a), (b) and (c) of Theorem 7 follow easily from Lemma 10.

Analyzing the different distributions of the particles on the central configurations described in Lemma 10, we see that each equilateral triangle gives two different classes of central configurations and an isosceles triangle gives six different classes of central configurations.

We remark that the equilateral triangle central configurations for $I>1 / 2$ bifurcate for $I=1 / 2$ from the circle of equilibrium points of the Lennard--Jones 3-body problem that we give in Theorem 6(b).

\subsection{Spectral stability of the Central Configurations}

Now we analyze the spectral stability of the relative equilibria associated to the central configurations given in Theorem 7 .

Let $\mathbf{x}=\left(\mathbf{x}_{1}, \mathbf{x}_{2}, \mathbf{x}_{3}\right)$ be a central configuration of the Lennard-Jones 3 -body problem. The relative equilibrium associated to $\mathbf{x}$ is spectrally stable if the $\lambda$ 's satisfying

$$
\operatorname{det}(B)=\operatorname{det}\left[-D \nabla U(\mathbf{x})+\left(\omega^{2}-\lambda^{2}\right) I d-2 \lambda \omega J\right]=0,
$$

are zero or purely imaginary (see Section 2 for details). Here

$$
U(\mathbf{x})=\frac{1}{r_{12}^{12}}-\frac{2}{r_{12}^{6}}+\frac{1}{r_{13}^{12}}-\frac{2}{r_{13}^{6}}+\frac{1}{r_{23}^{12}}-\frac{2}{r_{23}^{6}},
$$

with $r_{i j}=\left|\mathbf{x}_{i}-\mathbf{x}_{j}\right|, I d$ is the $6 \times 6$ identity matrix, and $\omega$ is given by (35) or (47) in the collinear or in the planar case, respectively.

In particular, we obtain the following result. Statements (a) and (c) are proved analytically. These two statements give the regions of spectral stability or instability of the relative equilibria associated to symmetrical central configurations, which are the more interesting from a physical point of view. We also give some numerical evidences of statements $\left(b^{*}\right),\left(d^{*}\right)$ and $\left(e^{*}\right)$.

THEOREM 11. The relative equilibria associated to central configurations of the planar Lennard-Jones 3-body problem given by Theorem 7 satisfy the following. 
(a) The relative equilibrium associated to a collinear equilateral central configuration is spectrally stable for $I \in\left[i_{2}, I_{6}\right]$, with $i_{2}=$ $\frac{1}{4}\left(\frac{5461}{85}\right)^{1 / 3}=1.00129 \ldots$, and unstable for $I \in\left(I_{4}, i_{2}\right) \cup\left(I_{6}, \infty\right)$.

$\left(b^{*}\right)$ The relative equilibrium associated to a collinear isosceles central configuration is unstable for $I \in\left(I_{6}, \infty\right)$.

(c) The relative equilibrium associated to an equilateral triangle central configuration is spectrally stable for $I \in\left(I_{1}, j_{2}\right]$, with $j_{2}=$ $1 /\left(2^{2 / 3}\right)=0.629961 \ldots$, and unstable for $I>j_{2}$.

$\left(d^{*}\right)$ The relative equilibrium associated to the isosceles triangle central configuration $T_{1}$ is unstable for $I \in\left(I_{2}, I_{5}\right)$.

$\left(e^{*}\right)$ The relative equilibrium associated to the isosceles triangle central configuration $T_{2}$ is spectrally stable for $I \in\left[k_{1}, k_{2}\right]=[0.6115 \ldots$, $0.6185 \ldots]$ and unstable for $I \in\left(I_{2}, k_{1}\right) \cup\left(k_{2}, \infty\right)$.

Theorem 11 is shown in Subsections 4.3.1 and 4.3.2.

In Figure 2 we have represented the regions of spectral stability for the relative equilibria associated to central configurations of the Lennard-Jones 3-body problem with a bold line.

\subsubsection{Collinear central configurations}

Collinear equilateral central configurations

We choose a representant $\mathbf{x}$ of the collinear equilateral central configurations (see Lemma 9(a)) as follows. Set $\mathbf{x}=\left(\mathbf{x}_{1}, \mathbf{x}_{2}, \mathbf{x}_{3}\right)$ with $\mathbf{x}_{1}=$ $(\sqrt{I}, 0), \mathbf{x}_{2}=(0,0)$ and $\mathbf{x}_{3}=(-\sqrt{I}, 0)$ and $I>I_{4}=\frac{1}{4}\left(\frac{2731}{43}\right)^{1 / 3}=$ $0.99745 \ldots$. For this central configuration we have that $a_{12}=a_{23}=\sqrt{I}$ and $a_{13}=2 \sqrt{I}$ (see Lemma 9(a) again). Thus, from (35) we have that

$$
\omega= \pm \frac{3}{32 \sqrt{2}} \sqrt{\frac{-2731+2752 I^{3}}{I^{7}}} .
$$

Then, solving (50) with respect to $\lambda$ we obtain the following solutions

$\lambda_{1}=0$, with multiplicity 2,

$\lambda_{2}= \pm \omega i$, with multiplicity 2 ,

$\lambda_{3}= \pm \frac{3}{32} \sqrt{\frac{-13655+5504 I^{3}}{I^{7}}}$,

$\lambda_{4}= \pm \sqrt{\frac{-417789}{2048 I^{7}}+\frac{3069}{32 I^{4}}-\frac{9 \sqrt{83966-89983 I^{3}+24512 I^{6}}}{8 \sqrt{2} I^{7}}}$, 


$$
\lambda_{5}= \pm \sqrt{\frac{-417789}{2048 I^{7}}+\frac{3069}{32 I^{4}}+\frac{9 \sqrt{83966-89983 I^{3}+24512 I^{6}}}{8 \sqrt{2} I^{7}}} .
$$

Now, we analyze if $\lambda_{i}$ is purely imaginary or real for $I \in\left(I_{4}, \infty\right)$ and for $i=3,4,5$.

Let $f_{3}(I)=-13655+5504 I^{3}$, then $f_{3}(I)=0$ if $I=i_{1}=\frac{1}{4}\left(\frac{13655}{86}\right)^{1 / 3}$ $=1.35375 \ldots, f_{3}(I)<0$ if $I \in\left(I_{4}, i_{1}\right)$, and $f_{3}(I)>0$ if $I \in\left(i_{1}, \infty\right)$.

It is easy to see that $83966-89983 I^{3}+24512 I^{6}>0$ for all $I$. Let

$$
f_{4}(I)=\frac{-417789}{2048 I^{7}}+\frac{3069}{32 I^{4}}-\frac{9 \sqrt{83966-89983 I^{3}+24512 I^{6}}}{8 \sqrt{2} I^{7}},
$$

and

$$
f_{5}(I)=\frac{-417789}{2048 I^{7}}+\frac{3069}{32 I^{4}}+\frac{9 \sqrt{83966-89983 I^{3}+24512 I^{6}}}{8 \sqrt{2} I^{7}} .
$$

We see that $f_{4}(I)<0$ for $I \in\left(I_{4}, \infty\right)$. Moreover $f_{5}(I)=0$ for $I=i_{2}=$ $\frac{1}{4}\left(\frac{5461}{85}\right)^{1 / 3}=1.00129 \ldots$ and $I=i_{3}=\frac{1}{4}\left(\frac{36409}{313}\right)^{1 / 3}=I_{6} ; f_{5}(I)>0$ for $I \in\left(I_{4}, i_{2}\right), f_{5}(I)<0$ for $I \in\left(i_{2}, i_{3}\right)$, and finally $f_{5}(I)>0$ for $I \in\left(i_{3}, \infty\right)$.

In short, we have proved the following result, which corresponds to statement (a) of Theorem 11.

LEMMA 12. The relative equilibrium solution associated to a collinear equilateral central configuration of the Lennard-Jones 3-body problem is spectrally stable for $I \in\left[i_{2}, I_{6}\right]$, and unstable for $I \in\left(I_{4}, i_{2}\right) \cup\left(I_{6}, \infty\right)$.

Collinear isosceles central configurations

We do not have an analytic expression for the collinear isosceles central configurations, so we cannot study their stability analytically. Nevertheless, we have numerical evidence that they are unstable because equation (50) has one solutions with positive real part and another one with negative real part. This shows statement $\left(\mathrm{b}^{*}\right)$ of Theorem 11. In Figure 6 we have plotted the two solutions of (50) with non-zero real part.

\subsubsection{Planar central configurations}

As usual, for a planar central configuration we understand a noncollinear central configuration.

Planar equilateral central configurations

We choose a representant $\mathbf{x}$ of the planar equilateral central configurations (see Subsection 4.2.2) as follows. Set $\mathbf{x}=\left(\mathbf{x}_{1}, \mathbf{x}_{2}, \mathbf{x}_{3}\right)$, with $\mathbf{x}_{1}=$ 


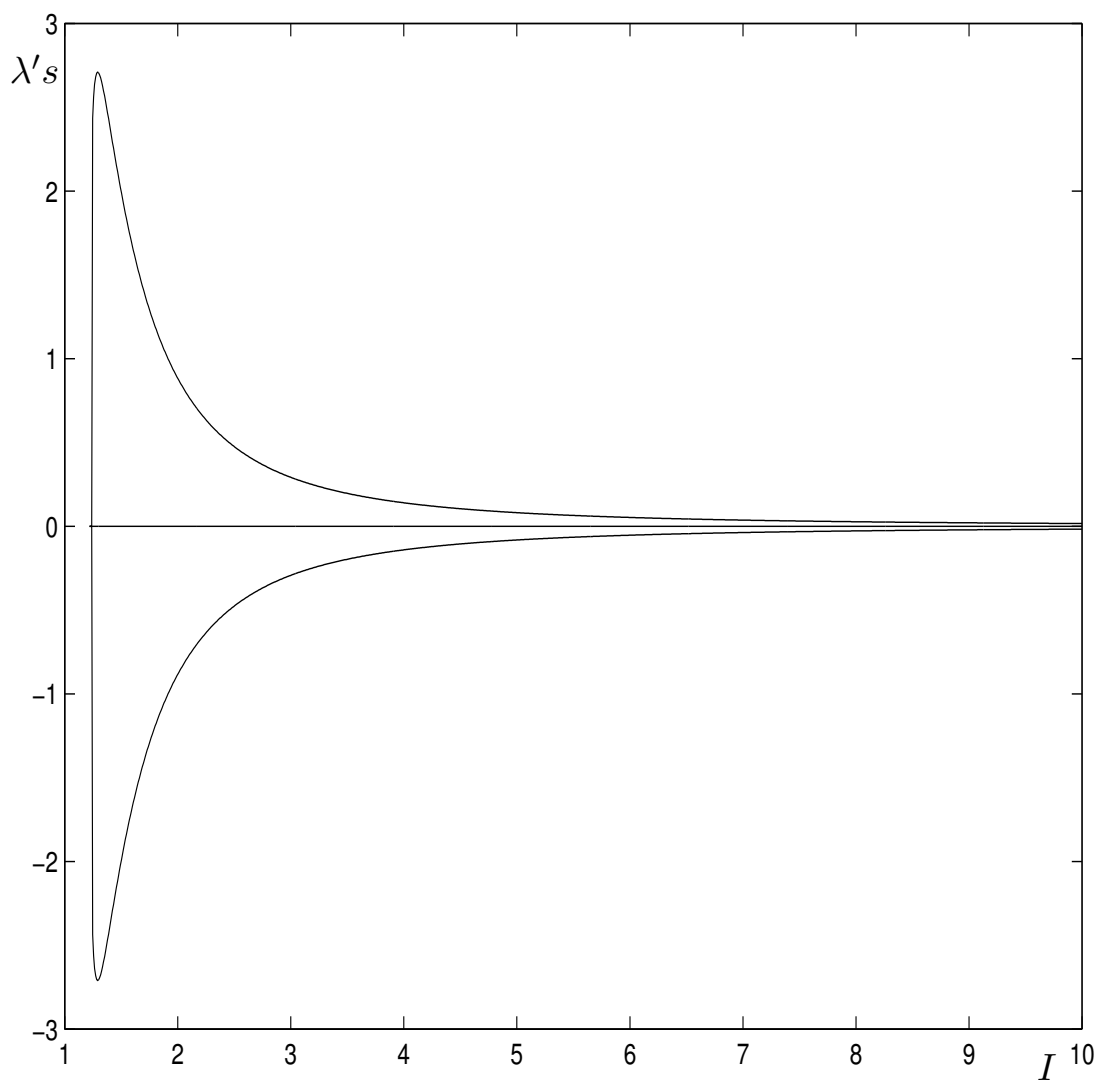

Figure 6. Real part of a solution of (50) for $I \in\left(I_{6}, 10\right)$ on the family of collinear isosceles central configurations.

$\left(\sqrt{\frac{2 I}{3}}, 0\right), \mathbf{x}_{2}=\left(-\frac{\sqrt{I}}{\sqrt{6}}, \frac{\sqrt{I}}{\sqrt{2}}\right)$ and $\mathbf{x}_{3}=\left(-\frac{\sqrt{I}}{\sqrt{6}},-\frac{\sqrt{I}}{\sqrt{2}}\right)$ and $I>I_{1}=1 / 2$. For this central configuration we have that $r_{12}=r_{13}=r_{23}=\sqrt{2 I}$. Thus, from (47), we have that

$$
\omega= \pm \frac{3}{4} \sqrt{\frac{-1}{2 I^{7}}+\frac{4}{I^{4}}} .
$$

Then, solving (50) with respect to $\lambda$, we obtain the following solutions

$\lambda_{1}=0$, with multiplicity 2,

$\lambda_{2}= \pm \omega i$, with multiplicity 2 ,

$\lambda_{3}= \pm \frac{3 \sqrt{-5+16 I^{3}}}{4 I^{7 / 2}}$,

$\lambda_{4}= \pm \sqrt{\frac{-45}{32 I^{7}}+\frac{9}{2 I^{4}}-\frac{9 \sqrt{3} \sqrt{-I^{14}+12 I^{17}-32 I^{20}}}{8 \sqrt{2} I^{14}}}$, 


$$
\lambda_{5}= \pm \sqrt{\frac{-45}{32 I^{7}}+\frac{9}{2 I^{4}}+\frac{9 \sqrt{3} \sqrt{-I^{14}+12 I^{17}-32 I^{20}}}{8 \sqrt{2} I^{14}}} .
$$

Now, we analyze if $\lambda_{i}$ is purely imaginary or real for $I \in\left(I_{1}, \infty\right)$ and for $i=3,4,5$.

Let $g_{3}(I)=-5+16 I^{3}$, then $g_{3}(I)=0$ if $I=j_{1}=\frac{1}{2}\left(\frac{5}{2}\right)^{1 / 3}=$ $0.678604 \ldots, g_{3}(I)<0$ if $I \in\left(I_{1}, j_{1}\right)$, and $g_{3}(I)>0$ if $I \in\left(j_{1}, \infty\right)$.

Let $g(I)=-I^{14}+12 I^{17}-32 I^{20}$,

$$
g_{4}(I)=\sqrt{\frac{-45}{32 I^{7}}+\frac{9}{2 I^{4}}-\frac{9 \sqrt{3} \sqrt{g(I)}}{8 \sqrt{2} I^{14}}},
$$

and

$$
g_{5}(I)=\sqrt{\frac{-45}{32 I^{7}}+\frac{9}{2 I^{4}}+\frac{9 \sqrt{3} \sqrt{g(I)}}{8 \sqrt{2} I^{14}}} .
$$

We see that $g(I)=0$ when $I=0, I=I_{1}$ and $I=j_{2}=1 /\left(2^{2 / 3}\right)=$ $0.629961 \ldots$, moreover $g(I)>0$ in $I \in\left(I_{1}, j_{2}\right)$, and $g(I)<0$ in $I \in$ $\left(j_{2}, \infty\right)$. On the other hand, $g_{4}(I)<0$ for $I \in\left(I_{1}, j_{2}\right], g_{4}(I)$ is complex for $I \in\left(j_{2}, \infty\right), g_{5}(I)=0$ for $I=j_{3}=\frac{7^{1 / 3}}{22^{2 / 3}}=0.602536 \ldots, g_{5}(I)<0$ for $I \in\left(I_{1}, j_{3}\right) \cup\left(j_{3}, j_{2}\right]$ and $g_{5}(I)$ is complex for $I \in\left(j_{2}, \infty\right)$.

In short, we have proved the following result, which corresponds to statement (c) of Theorem 11.

LEMMA 13. The relative equilibrium solution associated to an equilateral triangle central configuration of the Lennard-Jones 3-body problem is spectrally stable for $I \in\left(I_{1}, j_{2}\right]$ and unstable for $I>j_{2}$.

\section{Planar isosceles central configurations}

Similarly to the collinear isosceles central configurations, we do not have an analytic expression for the planar isosceles central configurations, so we have studied their stability numerically.

The planar isosceles triangle central configurations of type (iii) are unstable because (50) has one solution with positive real part and another one with negative real part. This shows statement $\left(\mathrm{d}^{*}\right)$ of Theorem 11. In Figure 7 we have plotted a solution of (50) with nonzero real parts. We note that for our convenience, in Figure 7, we have plotted a solution of (50) on the family of isosceles triangle central configurations of type (iii) as a function of $c$ instead $I$, where $c$ and $I$ are related by the continuous line in Figure 5.

The planar isosceles triangle central configurations of type (iv) in general are unstable, but there is a small region in which they are 


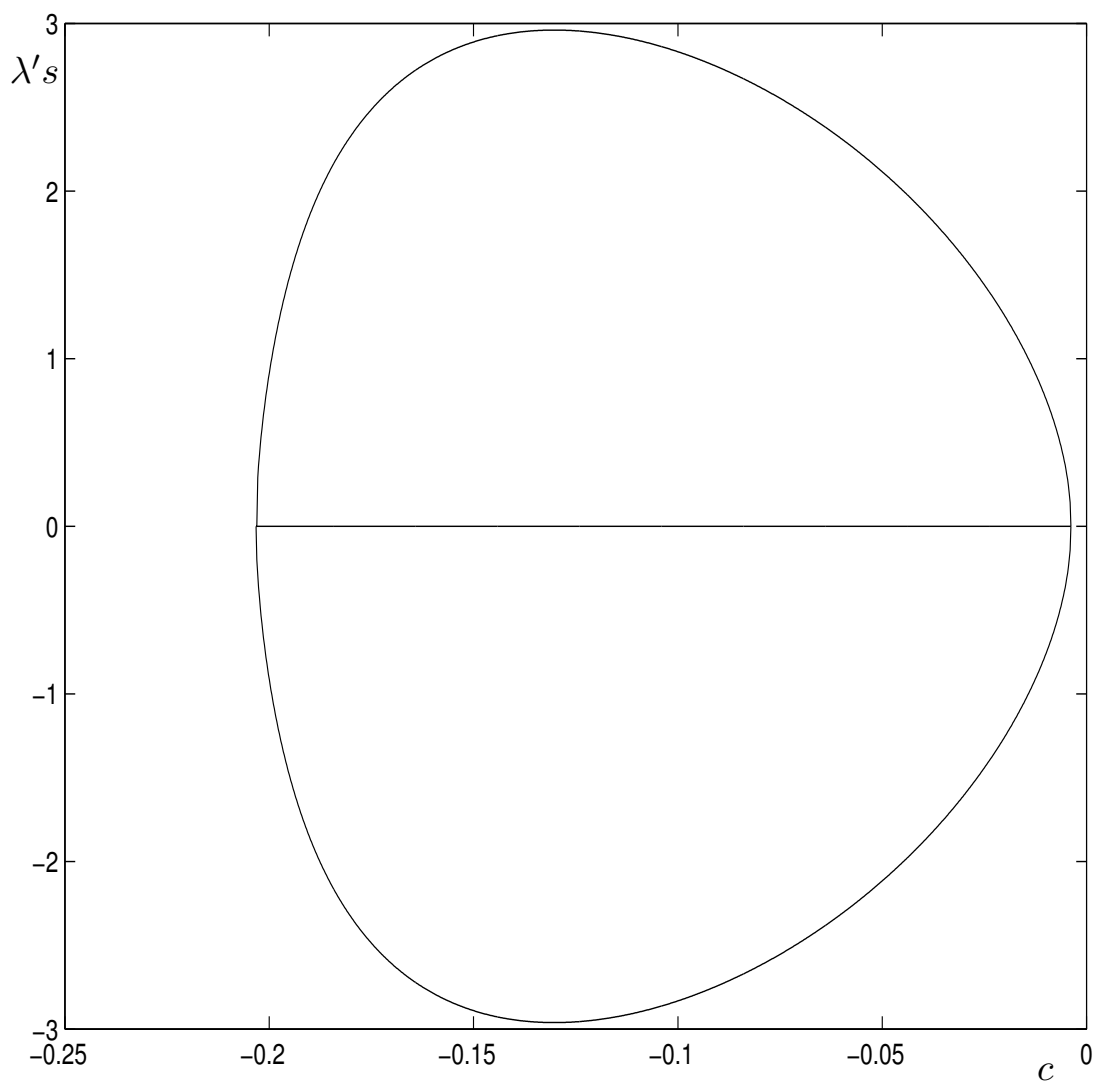

Figure 7. Real part of a solution of (50) on the family of isosceles triangle central configurations of type (iii).

spectrally stable. This shows statement $\left(\mathrm{e}^{*}\right)$ of Theorem 11. In Figure 8 we have plotted the solutions of $(50)$ with non-zero real parts as a function of $c$, where $c$ and $I$ are related by the dashed line in Figure 5. The region of spectral stability corresponds to $c \in\left[\gamma_{1}, \gamma_{2}\right]=$ $[-0.1996 \ldots,-0.1942 \ldots]$, or equivalently, to $I \in\left[k_{1}, k_{2}\right]=[0.6115 \ldots$, $0.6185 \ldots]$.

\section{Acknowledgements}

The first two authors are partially supported by the grants MCYT number BFM2002-04236-C02-02 and CIRIT number 2001SGR00173.

The third author was partially supported by CONACYT-México Grant number 32167-E. 


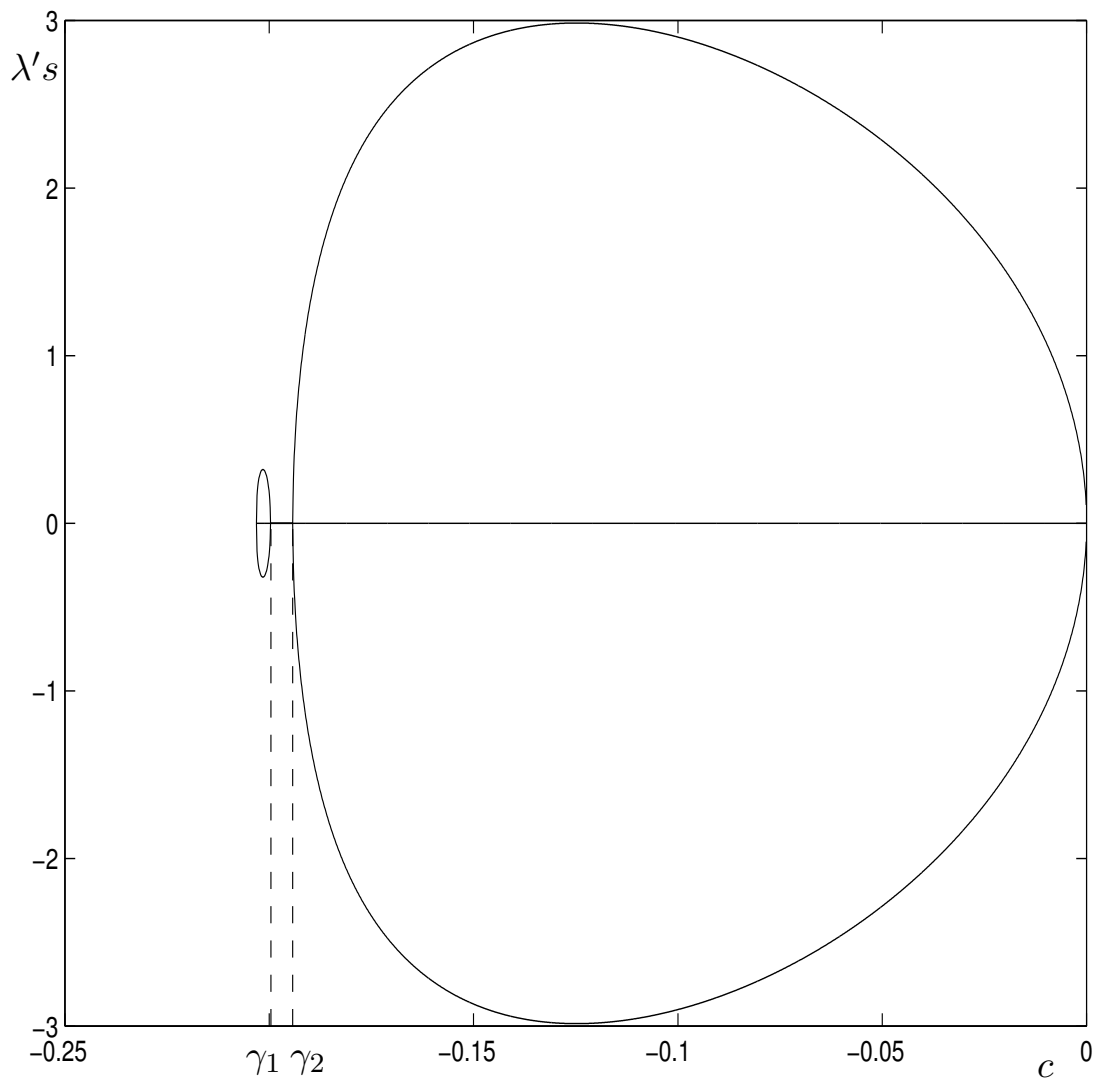

Figure 8. Real part of a solution of (50) on the family of isosceles triangle central configurations of type (iv).

\section{Appendix}

The goal of this appendix is to prove the next result.

PROPOSITION 14. Let $u=f(\mathbf{x})$ with $\mathbf{x}=\left(x_{1}, x_{2}, \ldots, x_{n}\right)$ and let $x_{1}=g_{1}(\mathbf{y}), x_{2}=g_{2}(\mathbf{y}), \ldots, x_{n}=g_{n}(\mathbf{y})$ with $\mathbf{y}=\left(y_{1}, y_{2}, \ldots, y_{m}\right)$ and $m \geqslant n$. If $\operatorname{rank}(A)=n$, with

$$
A=\left(\begin{array}{ccc}
\frac{\partial x_{1}}{\partial y_{1}} & \cdots & \frac{\partial x_{n}}{\partial y_{1}} \\
\vdots & \ddots & \vdots \\
\frac{\partial x_{1}}{\partial y_{m}} & \cdots & \frac{\partial x_{n}}{\partial y_{m}}
\end{array}\right)
$$

then $\nabla f(\mathbf{x})=\mathbf{0}$ if and only if $\nabla u(\mathbf{y})=\mathbf{0}$. 
Proof: We know that

$$
\frac{\partial u}{\partial y_{i}}=\frac{\partial f}{\partial x_{1}} \frac{\partial x_{1}}{\partial y_{i}}+\ldots+\frac{\partial f}{\partial x_{n}} \frac{\partial x_{n}}{\partial y_{i}}, \quad i=1, \ldots, m,
$$

then

$$
\nabla u(\mathbf{y})=A \nabla f(\mathbf{x}) .
$$

We consider (51) like a system of linear equations. It is clear, from (51), that if $\nabla f(\mathbf{x})=\mathbf{0}$, then $\nabla u(\mathbf{y})=\mathbf{0}$. The converse is only true when the $\operatorname{rank}(A)$ equals the number of unknowns; that is, if $\operatorname{rank}(A)=n$ which proves the result.

We note that if $m=n$, then Proposition 14 can be applied only when $x_{1}=g_{1}(\mathbf{y}), x_{2}=g_{2}(\mathbf{y}), \ldots, x_{n}=g_{n}(\mathbf{y})$ defines a change of variables. Finally, if $m>n$, Proposition 14 can be applied when $\operatorname{rank}(A)=n$, although $x_{1}=g_{1}(\mathbf{y}), x_{2}=g_{2}(\mathbf{y}), \ldots, x_{n}=g_{n}(\mathbf{y})$ does not define a change of variables.

\section{References}

Arnold, V.I.: 1978, Mathematical Methods of Classical Mechanics, Springer Verlag, Berlin.

Guckenheimer, J. and Holmes, P.: 1986, Nonlinear oscilations, dynamical systems, and bifurcations of vector fields, Springer Verlag, New-York.

Hoare, M.R. and Pal, P.: 1971, 'Statics and stability of small cluster nuclei', Nature Physical Science $\mathbf{2 3 0}$.

Meyer, K.R. and Hall, G.R.: 1991, An Introduction to Hamiltonian Dynamical Systems, Springer-Verlag, New York.

Moeckel, R.: 1995, 'Linear stability of some symmetrical classes of relative equilibria', In: Hamiltonian dynamical systems (Cincinnati, OH, 1992), IMA Vol. Math Appl., 63, Springer, New York, 291-317.

Moeckel, R.: 1994, 'Linear stability of relative equilibria with a dominant mass', $J$. Dynam. Differential Equations, 6, 37-51.

Wales, D.J. and Doye, J.P.K.: 1997, 'Global optimization by basin-hopping and the lowest energy structures of Lennard-Jones clusters containing up to 110 atoms', J. Phys. Chem. A, 101, 5111-5116. 
articleclp.tex; 25/02/2004; $17: 20 ;$ p.36 